\title{
Multifractal spectra of Birkhoff averages for a piecewise monotone interval map
}

by

\author{
Franz Hofbauer (Wien)
}

\begin{abstract}
We study the entropy spectrum of Birkhoff averages and the dimension spectrum of Lyapunov exponents for piecewise monotone transformations on the interval. In general, these transformations do not have finite Markov partitions and do not satisfy the specification property. We characterize these multifractal spectra in terms of the Legendre transform of a suitably defined pressure function.
\end{abstract}

1. Introduction. Multifractal analysis of dynamical systems studies the complexity of the level sets of local quantities, such as Birkhoff averages, local dimensions, and Lyapunov exponents. The complexity is usually measured either by topological entropy or by Hausdorff dimension. In the first case one gets the so called entropy spectrum of the local quantity, in the second case the dimension spectrum. For an introduction to this subject see [1] or [1]. For several classes of dynamical systems, which usually either have a finite Markov partition or satisfy the specification property, entropy and dimension spectra have been investigated. They are characterized either by a conditional variational principle or by the Legendre transform of a pressure function (see for instance [13], [14, [15], 2]).

The class of dynamical systems we consider here are piecewise monotone transformations on the interval $[0,1]$, which in general do not have a finite Markov partition and do not satisfy the specification property. We investigate the level sets of Birkhoff averages of regular functions $g:[0,1] \rightarrow \mathbb{R}$ and characterize their entropy and Hausdorff dimension in terms of the Legendre transform of a pressure function. In the case of Hausdorff dimension we do this only for the special case of Lyapunov exponents. We use similar methods to [7, where a multifractal spectrum of local dimensions is considered.

2010 Mathematics Subject Classification: 37E05, 37B40, 37D35.

Key words and phrases: piecewise monotone map, Birkhoff averages, Lyapunov exponent, multifractal spectra, Legendre transform, pressure function. 
A transformation $T:[0,1] \rightarrow[0,1]$ is called piecewise monotone if there is a partition $0=c_{0}<c_{1}<\cdots<c_{N}=1$ of $[0,1]$ such that $T \mid\left(c_{i-1}, c_{i}\right)$ is strictly monotone and continuous for $1 \leq i \leq N$. Furthermore, we assume that this partition is a generator, which means that $\bigcup_{j=0}^{\infty} T^{-j}\left\{c_{0}, c_{1}, \ldots, c_{N}\right\}$ is dense in $[0,1]$.

A closed subset $A$ of $[0,1]$ is called invariant if $T(A) \subset A$. A closed invariant subset $A$ is called completely invariant if $x \in A$ is equivalent to $T(x) \in A$ for all $x \in[0,1] \backslash P$. Throughout the paper we work on a fixed closed topologically transitive $T$-invariant subset $A$. It might be more natural for piecewise monotone transformations $T$ to use $T(A \backslash P) \subset A$ as the definition of an invariant subset, but in this case one can get $T(A) \subset A$ by redefining $T$ on the set $P$, provided $A$ is closed and topologically transitive. Therefore we use $T(A) \subset A$ as the definition of a $T$-invariant set.

The complexity of the level sets of Birkhoff averages is measured by topological entropy or by Hausdorff dimension. Since these level sets are not compact, we introduce a notion of topological entropy defined for any subset $Q$ of $[0,1]$ which we denote by $\operatorname{ent}_{\mathrm{B}}(Q)$. It is essentially Bowen's definition given in [3], very similar to the definition of Hausdorff dimension. We investigate this kind of entropy in Section 2.

Topological pressure is usually defined only for continuous transformations. Since we investigate transformations which are not continuous, we need a suitable definition of topological pressure. This is given in Section 3 . We say that a function $f:[0,1] \rightarrow \mathbb{R}$ is regular if $f_{+}(x):=\lim _{y \downarrow x} f(y)$ for $x \in[0,1)$ and $f_{-}(x):=\lim _{y \uparrow x} f(y)$ for $x \in(0,1]$ exist and if $f(x)$ lies between $f_{-}(x)$ and $f_{+}(x)$ for all $x \in(0,1)$. For a closed $T$-invariant subset $A$ and a regular function $f$ we define a pressure $q(T \mid A, f)$ by exhausting $A$ by subsets which have a Markov partition, and approximating $f$ by continuous functions from below. This is the definition used also in [7]. It is shown there that it coincides with the usual notion of pressure if $T$ and $f$ are continuous. This means that in the case where the pressure is usually defined, we do not change the notion of pressure.

Throughout this paper we shall assume that $T$ is a piecewise monotone transformation on $[0,1]$ and that the partition occurring in the definition of piecewise monotonicity is a generator. We consider a completely invariant topologically transitive closed subset $A$ of $([0,1], T)$ with $h_{\mathrm{top}}(T \mid A)>0$, and investigate the Birkhoff averages of a regular function $g:[0,1] \rightarrow \mathbb{R}$ on $A$. We set $S_{n} g=\sum_{j=0}^{n-1} g \circ T^{j}$ for $n \geq 1$ and define the level sets

$$
L_{a}=\left\{x \in A: \lim _{n \rightarrow \infty} \frac{1}{n} S_{n} g(x)=a\right\} \quad \text { for } a \in \mathbb{R} .
$$

More generally, for $u, v \in \mathbb{R}$ with $u \leq v$ we consider the sets

$$
L_{u, v}=\left\{x \in A: \liminf _{n \rightarrow \infty} \frac{1}{n} S_{n} g(x) \geq u, \limsup _{n \rightarrow \infty} \frac{1}{n} S_{n} g(x) \leq v\right\} .
$$


The level set $L_{a}$ is then the set $L_{a, a}$. The Legendre transform of the pressure function $\tau(s)=q(T \mid A, s g)$ is defined by

$$
\hat{\tau}(a)=\inf _{s \in \mathbb{R}}(\tau(s)-a s) .
$$

The set $H$ on which $\hat{\tau}$ is finite is a bounded closed interval (it is a single point if and only if $\tau$ is linear), and $\hat{\tau}$ is a concave function on $H$ (see Section 5). In Sections 4 and 7 we prove the following results (see Theorems 10 and 17): For $u$ and $v$ in $\mathbb{R}$ with $u \leq v$ and $H \cap[u, v] \neq \emptyset$ we have $\operatorname{ent}_{\mathrm{B}}\left(L_{u, v}\right)=\max _{a \in H \cap[u, v]} \hat{\tau}(a)$. In particular, $\operatorname{ent}_{\mathrm{B}}\left(L_{a}\right)=\hat{\tau}(a)$ for all $a \in H$. Furthermore, for each $a \in H$ with $\hat{\tau}(a)>0$ there is an ergodic invariant probability measure $\mu$ with $\mu\left(L_{a}\right)=1$ and $h_{\mu}=\hat{\tau}(a)$.

Since the pressure function involved in the above results is defined as a supremum over Markov subsets of $([0,1], T)$, we cannot show that Birkhoff averages are always inside $H$. But also no example is known for which Birkhoff averages are outside $H$. Nevertheless, we prove the following result in Section 7 Let $K$ be the set of all $x \in A$ such that all limit points of the sequence $n^{-1} S_{n} g(x)$ are less than the left or greater than the right endpoint of $H$. Then $\operatorname{ent}_{\mathrm{B}}(K)=0$.

Results of this kind are well known for certain classes of dynamical systems, which are conjugate to subshifts of finite type, in particular geometric constructions, repellers of smooth expanding maps, and locally maximal hyperbolic sets of diffeomorphisms. In [2] more general multifractal spectra are investigated. Results of [2] can also be applied to a piecewise monotone transformation $T$. By doubling a countable set of points in $[0,1]$ one gets a compact set $X$ on which $T$ is a continuous transformation. See Section 2 for the details of this construction. Since $T$ is expansive on $X$, Theorem 8 in [2] applies to the dynamical system $(X, T)$. As a special case one gets a formula for the entropy of the level sets of Birkhoff averages of a function $g$ as the Legendre transform of a pressure function, but only under the additional assumption that $g: X \rightarrow \mathbb{R}$ is continuous and has a unique equilibrium state.

The characterization of the dimension spectrum of Birkhoff averages by Legendre transforms works only in the special case of Lyapunov exponents. Set $\varphi=\log \left|T^{\prime}\right|$. For $x \in[0,1]$ the lower and the upper Lyapunov exponents are defined by

$$
\underline{\chi}(x)=\liminf _{n \rightarrow \infty} \frac{1}{n} S_{n} \varphi(x) \quad \text { and } \quad \bar{\chi}(x)=\limsup _{n \rightarrow \infty} \frac{1}{n} S_{n} \varphi(x) .
$$

If $\underline{\chi}(x)=\bar{\chi}(x)$, this common value is called the Lyapunov exponent at $x$ and denoted by $\chi(x)$. Again let $A$ be a completely invariant topologically transitive closed subset of $([0,1], T)$ with $h_{\text {top }}(T \mid A)>0$ and suppose that $\varphi=\log \left|T^{\prime}\right|$ is a regular function. For $u, v \in \mathbb{R}$ with $u \leq v$ we consider again the set $M_{u, v}=\{x \in A: u \leq \underline{\chi}(x) \leq \bar{\chi}(x) \leq v\}$. The level set 
$M_{a}$ which is the set of all $x \in A$ with $\chi(x)=a$ is then the set $M_{a, a}$. For $\tau(s)=q(T \mid A, s \varphi)$ define $H$ and $\hat{\tau}$ as above and set $\check{\tau}(a)=(1 / a) \hat{\tau}(a)$. Then $H$ is a bounded closed subinterval of $[0, \infty)$ (see Section 5). In Section 8 we prove the following result (see Theorem 20): For $u$ and $v$ in the interior of $H$ with $u \leq v$ we have $\operatorname{dim}_{H}\left(M_{u, v}\right)=\max _{a \in[u, v]} \check{\tau}(a)$. In particular, $\operatorname{dim}_{\mathrm{H}}\left(M_{a}\right)=\check{\tau}(a)$ for all $a \in \operatorname{int} H$.

This result has been shown in [4] under stronger assumptions. An invariant subset of a piecewise monotone interval map is considered there, which is conjugate to a mixing subshift of finite type and satisfies a distortion property and a weak expansion condition. Under such assumptions, further results are proved in [4, in particular sets of points with zero Lyapunov exponent are investigated.

We finish with some comments on the assumptions. One can split the nonwandering set of a piecewise monotone transformation $T$ into closed topologically transitive $T$-invariant subsets (see e.g. [5]). Those with nonzero topological entropy are often called basic sets. By the results of [5] a basic set can be written as $\bigcap_{i=0}^{\infty} \overline{F \backslash T^{-i} G}$ where $F$ and $G$ are $T$-invariant subsets which are finite unions of closed intervals and satisfy $G \subset F$. It is easy to see that this is a completely invariant subset for the transformation $T \mid F$, which is again a piecewise monotone interval map. Hence the above results hold for all basic sets of a piecewise monotone transformation.

We assume that the partition of $[0,1]$ into intervals on which $T$ is monotone is a generator. If this does not hold, then there is an interval $I$ such that $T^{n} \mid I$ is monotone for all $n$. We call a nondegenerate interval with this property an atomic interval. We have $\operatorname{ent}_{\mathrm{B}}(I)=0$ and $\operatorname{dim}_{\mathrm{H}}(I)=1$ for every atomic interval $I$. Therefore one cannot expect to characterize the multifractal spectrum by the Legendre transform of a pressure function if there are atomic intervals. In the case of the entropy spectrum we can consider all atomic intervals as single points. This modified dynamical system has a generator and we can apply the above result.

It does not matter how one defines the piecewise monotone transformation $T$ and the regular function $g$ at their points of discontinuity. The set $W$ of points whose orbits hit a point of discontinuity is countable. Changing $T$ or $g$ at a point of discontinuity causes the change of the level sets of Birkhoff averages of $g$ at most on the countable set $W$. Therefore, the entropy and Hausdorff dimension of the level sets do not change. Also the topological pressure, which we define for a regular function $f$, is not influenced by the values of $T$ and $f$ at their points of discontinuity.

2. Hausdorff dimension and entropy. We recall the definition of the Hausdorff dimension $\operatorname{dim}_{\mathrm{H}}(Q)$ of a set $Q \subset[0,1]$. For $\delta>0$ a finite or 
countable collection $\mathcal{C}$ of intervals of length $\leq \delta$ satisfying $Q \subset \bigcup_{C \in \mathcal{C}} C$ is called a $\delta$-cover of $Q$. Let $\Delta_{\delta}(Q)$ be the set of all $\delta$-covers of $Q$. For $t \in \mathbb{R}$ define

$$
\nu_{t}(Q)=\lim _{\delta \rightarrow 0} \inf _{\mathcal{C} \in \Delta_{\delta}(Q)} \sum_{C \in \mathcal{C}}|C|^{t}
$$

where $|C|$ denotes the length of the interval $C$. Then $\nu_{t}$ is an outer measure on $[0,1]$. There is $t_{0}$ such that $\nu_{t}(Q)=0$ for $t>t_{0}$ and $\nu_{t}(Q)=\infty$ for $t<t_{0}$. This $t_{0}$ is the Hausdorff dimension $\operatorname{dim}_{\mathrm{H}}(Q)$ of the set $Q$.

We need a notion of topological entropy for noncompact sets. We use a definition similar to that of Hausdorff dimension, which was introduced by Bowen in [3] (see also [12]). First we give a definition adapted to piecewise monotone transformations.

We call a finite set $\mathcal{Z}$ of open pairwise disjoint subintervals of $[0,1]$ a partition of $[0,1]$ if $[0,1] \backslash \bigcup_{Z \in \mathcal{Z}} Z$ is a finite set. We say that $T$ is piecewise monotone with respect to the partition $\mathcal{Z}$ if $T \mid Z$ is continuous and strictly monotone for all $Z \in \mathcal{Z}$.

Fix a partition $\mathcal{Z}$ with respect to which $T$ is piecewise monotone. Set $\mathcal{Z}_{j}=\bigvee_{i=0}^{j-1} T^{-i} \mathcal{Z}$ for $j \geq 1$ and $\mathcal{W}_{k}=\bigcup_{j=k}^{\infty} \mathcal{Z}_{j}$ for $k \geq 1$. For $Y \in \mathcal{W}_{1}$ let $\ell(Y)$ be the maximal $j$ such that $Y \in \mathcal{Z}_{j}$.

For $t \geq 0$ and $Q \subset[0,1]$ we define

$$
\gamma_{t}(Q)=\lim _{k \rightarrow \infty} \inf _{\mathcal{U} \in \Gamma_{k}(Q)} \sum_{Y \in \mathcal{U}} e^{-t \ell(Y)}
$$

where $\Gamma_{k}(Q)$ is the set of all finite or countable subsets $\mathcal{U}$ of $\mathcal{W}_{k}$ with $Q \subset$ $\bigcup_{Y \in \mathcal{U}} \bar{Y}$. It is easy to see that $\gamma_{t}$ defines an outer measure. Furthermore, for a subset $Q$ of $[0,1]$, there is $t_{0} \geq 0$ such that $\gamma_{t}(Q)=\infty$ for $t<t_{0}$ and $\gamma_{t}(Q)=0$ for $t>t_{0}$. We define $\operatorname{ent}_{\mathrm{B}}(Q)=t_{0}$ and call it the topological entropy of $Q$.

Since $\gamma_{t}$ is an outer measure for $t \geq 0$, it follows that $\operatorname{ent}_{\mathrm{B}}(S) \leq \operatorname{ent}_{\mathrm{B}}(Q)$ if $S \subset Q \subset[0,1]$, and $\operatorname{ent}_{\mathrm{B}}\left(\bigcup_{i=1}^{\infty} Q_{i}\right)=\sup _{i \geq 1} \operatorname{ent}_{\mathrm{B}}\left(Q_{i}\right)$ for any $Q_{i} \subset[0,1]$. Because ent $\operatorname{Ba}_{\mathrm{B}}(\{x\})=0$ for all $x \in[0,1]$ this implies that $\operatorname{ent}_{\mathrm{B}}(C)=0$ if $C$ is a countable set.

A partition $\mathcal{Z}$ with respect to which $T$ is piecewise monotone is not unique. The following two lemmas show that the above definition does not depend on the choice of $\mathcal{Z}$.

Lemma 1. Let $\mathcal{Z}$ be a partition with respect to which $T$ is piecewise monotone. If $J \subset[0,1]$ is an open interval such that $T^{j} \mid J$ is strictly monotone for $1 \leq j \leq k$, then $J$ has nonempty intersection with at most $k$ card $\mathcal{Z}$ intervals in $\mathcal{Z}_{k}$.

Proof. Let $0=c_{0}<c_{1}<\cdots<c_{N}=1$ be the endpoints of the intervals in $\mathcal{Z}$. We have $N=\operatorname{card} \mathcal{Z}$. Set $D=\left\{c_{1}, \ldots, c_{N-1}\right\}$. Then $\bigcup_{j=0}^{k-1}\left(T^{j} \mid J\right)^{-1} D$ 
are the endpoints of intervals in $\mathcal{Z}_{k}$ which fall into $J$. Their number is bounded by $k(N-1)$. Hence at most $k(N-1)+1$ intervals in $\mathcal{Z}_{k}$ have nonempty intersection with $J$. This proves the lemma, since $k(N-1)+1 \leq$ $k \operatorname{card} \mathcal{Z}$.

Lemma 2. Let $Q$ be a subset of $[0,1]$. Then $\operatorname{ent}_{\mathrm{B}}(Q)$ does not depend on the partition $\mathcal{Z}$ used in its definition, provided that $T$ is piecewise monotone with respect to $\mathcal{Z}$.

Proof. Let $\mathcal{Z}$ be a partition with respect to which $T$ is piecewise monotone and let $\Gamma_{k}(Q)$ and $\gamma_{t}(Q)$ be as above. Let $\tilde{\mathcal{Z}}$ be another partition with respect to which $T$ is piecewise monotone and let $\tilde{\Gamma}_{k}(Q)$ and $\tilde{\gamma}_{t}(Q)$ be as above, but for $\tilde{\mathcal{Z}}$ instead of $\mathcal{Z}$.

Let $t \geq 0$ and $\varepsilon>0$. There is a constant $\alpha_{t} \in(0, \infty)$ such that $e^{-(t+\varepsilon) n} n$ card $\tilde{\mathcal{Z}} \leq \alpha_{t} e^{-t n}$ for all $n \geq 1$. Fix $k$ and $\mathcal{U} \in \Gamma_{k}(Q)$. For $Y \in \mathcal{U}$ set

$$
\mathcal{K}_{Y}=\left\{Z \in \tilde{\mathcal{Z}}_{\ell(Y)}: Z \cap Y \neq \emptyset\right\} .
$$

Since $T^{j} \mid Y$ is strictly monotone for $1 \leq j \leq \ell(Y)$, it follows from Lemma 1 that $\operatorname{card} \mathcal{K}_{Y} \leq \ell(Y)$ card $\tilde{\mathcal{Z}}$. Let $\tilde{\ell}(Z)$ be the maximal $j$ such that $Z \in \tilde{\mathcal{Z}}_{j}$. Because $\mathcal{K}_{Y} \subset \tilde{\mathcal{Z}}_{\ell(Y)}$ we get $\tilde{\ell}(Z) \geq \ell(Y)$ for all $Z \in \mathcal{K}_{Y}$. This implies

$$
\sum_{Z \in \mathcal{K}_{Y}} e^{-(t+\varepsilon) \tilde{\ell}(Z)} \leq e^{-(t+\varepsilon) \ell(Y)} \ell(Y) \operatorname{card} \tilde{\mathcal{Z}} \leq \alpha_{t} e^{-t \ell(Y)} .
$$

Set $\tilde{\mathcal{U}}=\bigcup_{Y \in \mathcal{U}} \mathcal{K}_{Y}$. Since $\bar{Y} \subset \bigcup_{Z \in \mathcal{K}_{Y}} \bar{Z}$ and $\tilde{\ell}(Z) \geq \ell(Y) \geq k$ for all $Z \in \mathcal{K}_{Y}$, we get $\tilde{\mathcal{U}} \in \tilde{\Gamma}_{k}(Q)$. Summing the above estimate over $Y \in \mathcal{U}$ we get

$$
\sum_{Z \in \tilde{\mathcal{U}}} e^{-(t+\varepsilon) \tilde{\ell}(Z)} \leq \alpha_{t} \sum_{Y \in \mathcal{U}} e^{-t \ell(Y)} .
$$

Since $\mathcal{U} \in \Gamma_{k}(Q)$ was arbitrary this implies $\tilde{\gamma}_{t+\varepsilon}(Q) \leq \alpha_{t} \gamma_{t}(Q)$.

Now let $t_{0}$ be such that $\gamma_{t}(Q)=\infty$ for $t<t_{0}$ and $\gamma_{t}(Q)=0$ for $t>t_{0}$ and let $\tilde{t}_{0}$ be such that $\tilde{\gamma}_{t}(Q)=\infty$ for $t<\tilde{t}_{0}$ and $\tilde{\gamma}_{t}(Q)=0$ for $t>\tilde{t}_{0}$. The above inequality implies $\tilde{t}_{0} \leq t_{0}+\varepsilon$. Since $\varepsilon>0$ was arbitrary, we get $\tilde{t}_{0} \leq t_{0}$. The above proof with $\mathcal{Z}$ and $\tilde{\mathcal{Z}}$ interchanged gives $t_{0} \leq \tilde{t}_{0}$. Hence $t_{0}=\tilde{t}_{0}$.

This kind of definition of the entropy was given by Bowen in [3]. The sets considered there are subsets of topological dynamical systems. Since piecewise monotone transformations $T:[0,1] \rightarrow[0,1]$ may have discontinuities, we first construct continuous versions.

Fix a partition $\mathcal{Z}$ with respect to which $T$ is piecewise monotone. Here we can choose the coarsest such partition. Let $0=c_{0}<c_{1}<\cdots<c_{N}$ $=1$ be the finite set of the endpoints of the intervals in $\mathcal{Z}$ and set $W=$ $\bigcup_{i=0}^{\infty} T^{-i}\left\{c_{0}, c_{1}, \ldots, c_{N}\right\}$. We replace each $x \in W \backslash\{0,1\}$ by two points, $x-$ 
and $x+$. We denote this extended interval $[0,1]$ by $X$; it is compact with respect to the order topology. There is a continuous extension of $T$ from $[0,1] \backslash W$ to $X$, which we denote again by $T$. Now $(X, T)$ is a topological dynamical system and $\left\{\left[0, c_{1}-\right],\left[c_{1}+, c_{2}-\right], \ldots,\left[c_{N-1}-, 1\right]\right\}$ is a partition of $X$ into closed-open intervals, which we again denote by $\mathcal{Z}$.

Bowen's definition of the entropy of a subset $Q$ of $X$ is then as follows. Let $\mathcal{A}$ be a finite open cover of $X$. For $E \subset X$ let $m_{\mathcal{A}}(E)$ be the largest integer such that $T^{i}(E)$ is contained in some element of $\mathcal{A}$ for $0 \leq i<m_{\mathcal{A}}(E)$. Let $\tilde{\Gamma}_{\mathcal{A}, k}(Q)$ be the set of all finite or countable covers of $Q$ by arbitrary subsets $E$ of $X$ satisfying $m_{\mathcal{A}}(E) \geq k$. For $t \geq 0$ define

$$
\beta_{\mathcal{A}, t}(Q)=\lim _{k \rightarrow \infty} \inf _{\mathcal{E} \in \tilde{\Gamma}_{\mathcal{A}, k}(Q)} \sum_{E \in \mathcal{E}} e^{-t m_{\mathcal{A}}(E)} \quad \text { and } \quad \beta_{t}(Q)=\sup _{\mathcal{A}} \beta_{\mathcal{A}, t}(Q)
$$

where the supremum is taken over all finite open covers $\mathcal{A}$ of $X$. The entropy of $Q$ is then defined in [3] as the nonnegative number $h(Q)$ such that $\beta_{t}(Q)$ is $\infty$ for $t<h(Q)$ and 0 for $t>h(Q)$.

The following lemma shows that Bowen's definition of entropy given in [3] coincides with the definition we use in this paper. The complements of the set $[0,1] \backslash W$ in $[0,1]$ and in $X$ are countable and therefore have entropy zero. Hence it suffices to consider a subset $Q$ of $[0,1] \backslash W$, which is then a subset of $[0,1]$ and of $X$.

Lemma 3. For a subset $Q$ of $[0,1] \backslash W$ we have $h(Q)=\operatorname{ent}_{\mathrm{B}}(Q)$.

Proof. Let $\mathcal{Z}$ be the partition we have used in the definition of $X$. Let $\mathcal{A}$ be a finite open cover of $X$. Since $\mathcal{Z}$ is a generator, $W$ is dense in $[0,1]$. Therefore, choosing partition points in $W$, we find a partition $\mathcal{Y}$ of $[0,1]$ with respect to which $T$ is piecewise monotone and such that the corresponding partition of $X$ is also an open cover of $X$, which refines $\mathcal{A}$. By Lemma 2 we can use $\mathcal{Y}$ instead of $\mathcal{Z}$ in the definition of ent ${ }_{\mathrm{B}}$. If $\mathcal{U} \in \Gamma_{k}(Q)$, then $Q \subset \bigcup_{Y \in \mathcal{U}} Y$, since $Q \subset[0,1] \backslash W$ and the endpoints of intervals in $\mathcal{U}$ are contained in $W$, and $m_{\mathcal{A}}(Y) \geq m_{\mathcal{Y}}(Y)=\ell(Y) \geq k$ for all $Y \in \mathcal{U}$, since $\mathcal{Y}$ refines $\mathcal{A}$. This implies $\mathcal{U} \in \tilde{\Gamma}_{\mathcal{A}, k}(Q)$ and $\sum_{Y \in \mathcal{U}} e^{-t m_{\mathcal{A}}(Y)} \leq \sum_{Y \in \mathcal{U}} e^{-t \ell(Y)}$ for $t \geq 0$. Since $\mathcal{U} \in \Gamma_{k}(Q)$ was arbitrary, we get $\beta_{\mathcal{A}, t}(Q) \leq \gamma_{t}(Q)$ for $t \geq 0$. Since $\mathcal{A}$ was an arbitrary finite open cover of $X$, we have $\beta_{t}(Q) \leq \gamma_{t}(Q)$ for $t \geq 0$. This implies $h(Q) \leq \operatorname{ent}_{\mathrm{B}}(Q)$.

To show the opposite inequality we prove $\beta_{\mathcal{Z}, t}(Q) \geq \gamma_{t}(Q)$ where we consider $\mathcal{Z}$ as a finite open cover of $X$. Let $\mathcal{V} \in \tilde{\Gamma}_{\mathcal{Z}, k}(Q)$. For $E \in \mathcal{V}$ choose $Y_{E} \in \mathcal{Z}_{m_{\mathcal{Z}}(E)}$ with $E \cap Q \subset Y_{E}$. Then $\ell\left(Y_{E}\right) \geq m_{\mathcal{Z}}(E)$. With $\mathcal{U}=\left\{Y_{E}: E \in \mathcal{V}\right\}$ we have $\sum_{E \in \mathcal{V}} e^{-t m_{\mathcal{Z}}(E)} \geq \sum_{Y \in \mathcal{U}} e^{-t \ell(Y)}$ for all $t \geq 0$. Because $\mathcal{U} \in \Gamma_{k}(Q)$ and $\mathcal{V} \in \tilde{\Gamma}_{\mathcal{Z}, k}(Q)$ was arbitrary, we get $\beta_{\mathcal{Z}, t}(Q) \geq$ $\gamma_{t}(Q)$ and hence also $\beta_{t}(Q) \geq \gamma_{t}(Q)$ for all $t \geq 0$. This implies $h(Q) \geq$ $\operatorname{ent}_{\mathrm{B}}(Q)$. 
We state the following two lemmas in the form we need in this paper, and not in their strongest possible versions.

Lemma 4. Let $Q \subset[0,1]$ and let $\mu$ be an ergodic $T$-invariant probability measure on $[0,1]$. If $Q$ contains a subset of $\mu$-measure one, then $h_{\mu} \leq \operatorname{ent}_{\mathrm{B}}(Q)$.

Proof. If $h_{\mu}=0$ there is nothing to show. If $h_{\mu}>0$ then $\mu$ assigns measure zero to single points and $Q$ has a subset $D$ with $\mu(D)=1$ and $D \cap W=\emptyset$. Hence $D$ can be considered as a subset of $X$, and $\mu$ can be considered as an invariant probability measure on $(X, T)$. By Theorem 1 in [3] we get $h_{\mu} \leq \operatorname{ent}_{\mathrm{B}}(D)$. This implies $h_{\mu} \leq \operatorname{ent}_{\mathrm{B}}(Q)$.

For a probability measure $\mu$ on $[0,1]$ one defines the Hausdorff dimension $\operatorname{dim}_{\mathrm{H}}(\mu)$ as follows:

$\operatorname{dim}_{\mathrm{H}}(\mu)=\inf \left\{\operatorname{dim}_{\mathrm{H}}(B): B\right.$ a Borel subset of $[0,1]$ with $\left.\mu(B)=1\right\}$.

Then we have a lemma similar to the one above.

Lemma 5. Let $Q \subset[0,1]$. Suppose that $\varphi=\log \left|T^{\prime}\right|$ is a regular function and let $\mu$ be an ergodic T-invariant probability measure on $[0,1]$ with $h_{\mu}>0$. If $Q$ contains a Borel subset of $\mu$-measure one, then $h_{\mu} / \mu(\varphi) \leq \operatorname{dim}_{H}(Q)$.

Proof. By Theorem 2 in [6] we get $\mu(\varphi)>0$. We can apply Theorem 1 of [8] under the present assumptions (see the remark after Corollary 1 in [8]). This gives $\operatorname{dim}_{H}(\mu)=h_{\mu} / \mu(\varphi)$. By the definition of $\operatorname{dim}_{H}(\mu)$ we have $\operatorname{dim}_{\mathrm{H}}(Q) \geq \operatorname{dim}_{\mathrm{H}}(\mu)$ and the lemma is proved.

3. Pressure. Topological pressure is usually defined for continuous transformations and continuous functions on compact metric spaces. Since the transformations and functions we consider may have discontinuities, we give a suitable definition of pressure for a closed invariant subset $A$ of $([0,1], T)$ and a regular function $f$. We do this by approximation from below.

A closed subset $B$ of $[0,1]$ is called a Markov subset if there exists a partition $\mathcal{Y}$ of $[0,1]$ with respect to which $T$ is piecewise monotone with $T(B \cap Y) \subset B$ for all $Y \in \mathcal{Y}$ and such that for any two elements $Y_{1}$ and $Y_{2}$ of $\mathcal{Y}$ one has either $T\left(B \cap Y_{1}\right) \cap Y_{2}=\emptyset$ or $T\left(B \cap Y_{1}\right) \supset B \cap Y_{2}$. We then say that $\mathcal{Y}$ is a Markov partition for $B$.

For a Markov subset $B$ with Markov partition $\mathcal{Y}$ and a regular function $f:[0,1] \rightarrow \mathbb{R}$ we define the pressure as follows. Set $\mathcal{Y}_{n}=\bigvee_{i=0}^{n-1} T^{-i} \mathcal{Y}$ and $\mathcal{Y}_{n}(B)=\left\{B \cap Y \neq \emptyset: Y \in \mathcal{Y}_{n}\right\}$. Then we define

$$
p(T \mid B, f)=\lim _{n \rightarrow \infty} \frac{1}{n} \log \sum_{Y \in \mathcal{Y}_{n}(B)} \sup _{Y} e^{S_{n} f} .
$$

Let $A$ be a closed $T$-invariant subset and let $\mathcal{M}(A)$ be the set of all Markov 
subsets of $A$. We assume that $\mathcal{M}(A)$ is not empty. For a regular function $f:[0,1] \rightarrow \mathbb{R}$ let $\mathcal{C}(f)$ be the set of all continuous functions $h:[0,1] \rightarrow \mathbb{R}$ satisfying $h \leq f$. We define

$$
q(T \mid A, f)=\sup _{B \in \mathcal{M}(A)} \sup _{h \in \mathcal{C}(f)} p(T \mid B, h) .
$$

This can be written as $q(T \mid A, f)=\sup _{B \in \mathcal{M}(A)} q(T \mid B, f)$, since for a Markov subset $B$ we have $q(T \mid B, f)=\sup _{h \in \mathcal{C}(f)} p(T \mid B, h)$, because $B$ itself is an element of $\mathcal{M}(B)$. The following elementary properties are easy consequences of the definition.

Lemma 6. Let $A$ be a closed invariant subset with $\mathcal{M}(A) \neq \emptyset$ and let $f$ and $g$ be regular functions. Then $q(T \mid A, f+c)=q(T \mid A, f)+c$ for all $c \in \mathbb{R}$, and if $f \leq g$, then $q(T \mid A, f) \leq q(T \mid A, g)$.

A partition $\mathcal{Z}$ with respect to which $T$ is piecewise monotone is not unique. Let $A$ be a closed $T$-invariant subset of $[0,1]$ and let $\mathcal{Z}$ be a partition with respect to which $T$ is piecewise monotone. Let $\mathcal{M}(A, \mathcal{Z})$ be the set of all $B \in \mathcal{M}(A)$ which allow a Markov partition $\mathcal{Y}$ refining $\mathcal{Z}$, and define

$$
q_{\mathcal{Z}}(T \mid A, f)=\sup _{B \in \mathcal{M}(A, \mathcal{Z})} \sup _{h \in \mathcal{C}(f)} p(T \mid B, h) .
$$

In this paper we consider a topologically transitive completely invariant closed subset $A$ of $([0,1], T)$ with $h_{\mathrm{top}}(T \mid A)>0$. Then $\mathcal{M}(A, \mathcal{Z})$ is not empty. This can be shown using the so-called Markov diagram of the piecewise monotone transformation $T$ with respect to the partition $\mathcal{Z}$ (see [5]), which is a directed graph $(\mathcal{D}, \rightarrow)$ with countable vertex set $\mathcal{D}$, whose paths represent the orbits of $([0,1], T)$. As explained in Section 4 of [7], the topologically transitive completely invariant subset $A$ corresponds to an irreducible subgraph $(\mathcal{A}, \rightarrow)$ of $(\mathcal{D}, \rightarrow)$, which considered as a 0 -1-matrix has spectral radius larger than 1 due to $h_{\text {top }}(T \mid A)>0$ (see Section 3 in [5]). By the results in Chapter II of [5] there is a finite subset $\mathcal{B}$ of $\mathcal{A}$ such that the subgraph $(\mathcal{B}, \rightarrow)$ still has spectral radius larger than 1 . The set of all points in $A$ whose orbits are represented by paths in $(\mathcal{B}, \rightarrow)$ is then a Markov subset of $A$. In this way one gets $\mathcal{M}(A, \mathcal{Z}) \neq \emptyset$ and hence also $\mathcal{M}(A) \neq \emptyset$. So $q(T \mid A, f)$ and $q_{\mathcal{Z}}(T \mid A, f)$ are well defined.

Further results which we shall need in this paper and which are proved in Section 4 of [7] using the Markov diagram are collected in the following lemma.

Lemma 7. Let $A$ be a topologically transitive completely invariant closed subset of $([0,1], T)$ with $h_{\mathrm{top}}(T \mid A)>0$ and let $\mathcal{Z}$ be a partition with respect to which $T$ is piecewise monotone. Then $h_{\mathrm{top}}(T \mid A)=q(T \mid A, 0)$ and $q_{\mathcal{Z}}(T \mid A, f)=q(T \mid A, f)$ for all regular functions $f:[0,1] \rightarrow \mathbb{R}$. Furthermore, for regular functions $f_{1}, \ldots, f_{k}$ and $\varepsilon>0$ there is a topologically transitive 
subset $C \in \mathcal{M}(A, \mathcal{Z})$ with $h_{\mathrm{top}}(T \mid C)>0$ such that $q\left(T \mid A, f_{i}\right)<q\left(T \mid C, f_{i}\right)+\varepsilon$ for $1 \leq i \leq k$.

Next we show a kind of variational principle for the pressure introduced above. For a Markov subset $B$ and a regular function $f$ let $\mathcal{F}(B, f)$ be the set of all ergodic invariant probability measures on $B$ which assign measure zero to discontinuity points of $T$ and $f$, and let $\mathcal{F}(B)$ be the set of all ergodic invariant probability measures on $B$ which assign measure zero to all single points. For a completely invariant closed subset $A$ of $([0,1], T)$ and a partition $\mathcal{Z}$ with respect to which $T$ is piecewise monotone, set

$$
\mathcal{E}(A, \mathcal{Z}, f)=\bigcup_{B \in \mathcal{M}(A, \mathcal{Z})} \mathcal{F}(B, f) \text { and } \mathcal{E}(A, \mathcal{Z})=\bigcup_{B \in \mathcal{M}(A, \mathcal{Z})} \mathcal{F}(B) \text {. }
$$

Lemma 8. Let $f$ be a regular function. For a Markov subset $B$ of $([0,1], T)$ we have $q(T \mid B, f) \geq \sup _{\mu \in \mathcal{F}(B, f)}\left(h_{\mu}+\mu(f)\right)$. For a topologically transitive completely invariant closed subset $A$ of $([0,1], T)$ with $h_{\mathrm{top}}(T \mid A)>0$ and a partition $\mathcal{Z}$ with respect to which $T$ is piecewise monotone, we have

$$
q(T \mid A, f)=\sup _{\mu \in \mathcal{E}(A, \mathcal{Z}, f)}\left(h_{\mu}+\mu(f)\right)=\sup _{\mu \in \mathcal{E}(A, \mathcal{Z})}\left(h_{\mu}+\mu(f)\right) .
$$

Proof. Let $B$ be a Markov subset. Let $\mu \in \mathcal{F}(B, f)$. Then there are $h_{n} \in \mathcal{C}(f)$ with $h_{n}(x) \uparrow f(x)$ for all $x$ where $f$ is continuous. Since $\mu$ assigns measure zero to all points where $f$ is discontinuous, it follows that $\lim _{n \rightarrow \infty} \mu\left(h_{n}\right)=\mu(f)$. By the variational principle we have $h_{\mu}+\mu\left(h_{n}\right) \leq$ $p\left(T \mid B, h_{n}\right)$ for all $n$, which implies $h_{\mu}+\mu(f) \leq q(T \mid B, f)$. Since $\mu \in \mathcal{F}(B, f)$ was arbitrary,

$$
q(T \mid B, f) \geq \sup _{\mu \in \mathcal{F}(B, f)}\left(h_{\mu}+\mu(f)\right) .
$$

This is the first part of the lemma.

Let $B$ be a topologically transitive Markov subset with $h_{\text {top }}(T \mid B)>0$. Choose $\varepsilon>0$. There is a function $h \in \mathcal{C}(f)$ with $q(T \mid B, f)-\varepsilon<p(T \mid B, h)$. Since $h$ is continuous, there is $k \geq 1$ and a function $h: B \rightarrow \mathbb{R}$ which is constant on all cylinder sets of length $k$ and satisfies $\tilde{h} \leq h$ and $q(T \mid B, f)-$ $\varepsilon<p(T \mid B, \tilde{h})$. Since $B$ is a topologically transitive Markov subset with $h_{\text {top }}(T \mid B)>0$, there is an ergodic equilibrium state for the function $\tilde{h}$ on the Markov shift conjugate to $(B, T \mid B)$, which is a Markov measure with full support (see the proof of Lemma 4.7 in [16]). It assigns measure zero to single points and can therefore be considered as an invariant probability measure $\nu$ on $(B, T \mid B)$. We have

$$
q(T \mid B, f)-\varepsilon<p(T \mid B, \tilde{h})=h_{\nu}+\nu(\tilde{h}) \leq h_{\nu}+\nu(f) .
$$

Because $\nu \in \mathcal{F}(B)$ and $\varepsilon>0$ was arbitrary, we get

$$
q(T \mid B, f) \leq \sup _{\mu \in \mathcal{F}(B)}\left(h_{\mu}+\mu(f)\right) .
$$


Now let $A$ be a topologically transitive completely invariant closed subset of $([0,1], T)$ with $h_{\text {top }}(T \mid A)>0$ and let $\mathcal{Z}$ be a partition with respect to which $T$ is piecewise monotone. Using Lemma 7 and (3.1) we get

$$
q(T \mid A, f)=q_{\mathcal{Z}}(T \mid A, f)=\sup _{B \in \mathcal{M}(A, \mathcal{Z})} q(T \mid B, f) \geq \sup _{\mu \in \mathcal{E}(A, \mathcal{Z}, f)}\left(h_{\mu}+\mu(f)\right) .
$$

Let $\tilde{\mathcal{M}}(A, \mathcal{Z})$ be the set of all $B \in \mathcal{M}(A, \mathcal{Z})$ which are topologically transitive and satisfy $h_{\text {top }}(T \mid B)>0$. We get $q(T \mid A, f)=\sup _{B \in \tilde{\mathcal{M}}(A, \mathcal{Z})} q(T \mid B, f)$ again by Lemma 7, and together with (3.2) this implies

$$
q(T \mid A, f) \leq \sup _{B \in \tilde{\mathcal{M}}(A, \mathcal{Z})} \sup _{\mu \in \mathcal{F}(B)}\left(h_{\mu}+\mu(f)\right) \leq \sup _{\mu \in \mathcal{E}(A, \mathcal{Z})}\left(h_{\mu}+\mu(f)\right) .
$$

Since $\mathcal{E}(A, \mathcal{Z}) \subset \mathcal{E}(A, \mathcal{Z}, f)$ by definition, this completes the proof of the second part of the lemma.

We shall need Lemma 8 for the following two purposes: The equality $q(T \mid A, f)=\sup _{\mu \in \mathcal{E}(A, \mathcal{Z}, f)}\left(h_{\mu}+\mu(f)\right)$ shows that the pressure which we use in this paper, and the pressure used in [9], are the same. And using the equality $q(T \mid A, f)=\sup _{\mu \in \mathcal{E}(A, \mathcal{Z})}\left(h_{\mu}+\mu(f)\right)$ one easily shows that the map $f \mapsto q(T \mid A, f)$ is convex on the set of regular functions.

Finally, we mention that all theorems in this paper hold if we assume that $A$ is a Markov subset, instead of assuming that $A$ is completely invariant. This follows, since only the three lemmas of this section are needed in subsequent proofs, and it is easily seen that these lemmas also hold for a Markov subset $A$ instead of a completely invariant subset $A$.

4. Lower bounds. For a topologically transitive completely invariant closed subset $A$ of $([0,1], T)$ with $h_{\text {top }}(T \mid A)>0$ and a regular function $g:[0,1] \rightarrow \mathbb{R}$ we define

$$
\tau(s)=q(T \mid A, s g) \quad \text { for } s \in \mathbb{R} .
$$

The map $\tau: \mathbb{R} \rightarrow \mathbb{R}$ is convex, since it is the supremum of linear functions by Lemma 8. Set $H=\left\{a \in \mathbb{R}: \inf _{s \in \mathbb{R}}(\tau(s)-a s)>-\infty\right\}$, which is a nonempty interval, since $\tau$ is a convex function. We consider the Legendre transform $\hat{\tau}: H \rightarrow \mathbb{R}$ of $\tau$ defined by

$$
\hat{\tau}(a)=\inf _{s \in \mathbb{R}}(\tau(s)-a s) .
$$

In order to get lower bounds for topological entropy and Hausdorff dimension, we construct suitable ergodic invariant probability measures.

Proposition 9. Let $g:[0,1] \rightarrow \mathbb{R}$ be a regular function and let $A$ be a completely invariant topologically transitive closed subset of $([0,1], T)$ with $h_{\text {top }}(T \mid A)>0$. Then for every $a \in H$ we have $\hat{\tau}(a) \geq 0$ and there is a 
sequence $\left(\mu_{k}\right)_{k \geq 1}$ of ergodic invariant probability measures on A satisfying $\lim _{k \rightarrow \infty} \mu_{k}(g)=a$ and $\lim _{k \rightarrow \infty} h_{\mu_{k}}=\hat{\tau}(a)$.

Proof. First we construct differentiable approximations of $\tau$. To this end fix $\delta>0$ and let $F$ be a finite subset of $\mathbb{R}$. Choose $\alpha \in(0, \delta)$ such that $|\alpha s|<\delta$ for all $s \in F$ and let $\tilde{g}$ be a step function satisfying $g-\alpha<\tilde{g} \leq$ $g$. This is possible, since $g$ is a regular function. Using Lemma 6 we get $|q(T \mid A, s g)-q(T \mid A, s \tilde{g})|<\delta$ for all $s \in F$. Let $\mathcal{Z}$ be a partition of $[0,1]$ with respect to which $T$ is piecewise monotone and such that $\tilde{g}$ is constant on each element of $\mathcal{Z}$. By Lemma 7 there exists a topologically transitive $B \in \mathcal{M}(A, \mathcal{Z})$ with $h_{\text {top }}(T \mid B)>0$ such that $|q(T \mid A, s g)-q(T \mid B, s \tilde{g})|<\delta$ for all $s \in F$. Set $\tilde{\tau}(s)=q(T \mid B, s \tilde{g})$. We have shown that for each finite subset $F$ of $\mathbb{R}$ and each $\delta>0$ there are a topologically transitive set $B \in \mathcal{M}(A)$ with $h_{\text {top }}(T \mid B)>0$ and a step function $\tilde{g}$ which is constant on each element of a Markov partition $\mathcal{Y}$ of $B$ such that

$$
g-\delta<\tilde{g} \leq g \quad \text { and } \quad|\tau(s)-\tilde{\tau}(s)|<\delta \quad \text { for all } s \in F .
$$

Let $K$ be the transition matrix of the Markov map $T \mid B$ with respect to the Markov partition $\mathcal{Y}$. Then $K$ is irreducible, as $B$ is topologically transitive, and not a permutation matrix, as $h_{\mathrm{top}}(T \mid B)>0$. For $s \in \mathbb{R}$ let $M(s)$ be the matrix one gets if one multiplies the $Y$ th column of $K$ by the value of the function $e^{s \tilde{g}}$ at $Y$ for all $Y \in \mathcal{Y}$. As in the proof of Lemma 4.7 in [16] one gets an equilibrium state for the function $s \tilde{g}$ on the Markov shift conjugate to $(B, T \mid B)$, which is a Markov measure. It is determined by a left and a right eigenvector of $M(s)$. As $K$ is irreducible and not a permutation matrix, this equilibrium state is unique and ergodic and has full support and nonzero entropy (uniqueness follows as in Section 4 of [10]). It assigns measure zero to single points and can therefore be considered as a probability measure on $(B, T \mid B)$, which we denote by $\nu_{s}$. We have

$$
p(T \mid B, s \tilde{g})=h_{\nu_{s}}+\nu_{s}(s \tilde{g}) .
$$

Lemma 8 implies $h_{\nu_{s}}+\nu_{s}(u \tilde{g}) \leq q(T \mid B, u \tilde{g})$ for all $u \in \mathbb{R}$, since $\nu_{s}$ assigns measure zero to single points. Because of $q(T \mid B, s \tilde{g}) \leq p(T \mid B, s \tilde{g})$ and 4.2 . we have equality for $u=s$ and we get $p(T \mid B, s \tilde{g})=q(T \mid B, s \tilde{g})=\tilde{\tau}(s)$. Since $p(T \mid B, s \tilde{g})$ is the logarithm of the spectral radius of $M(s)$, which is a differentiable function in $s$, and since $u \mapsto h_{\nu_{s}}+u \nu_{s}(\tilde{g})$ is the tangent to this differentiable function at the point $s$ by the above results, it follows that

$$
\tilde{\tau}(s)=p(T \mid B, s \tilde{g}) \text { is differentiable and } \quad \tilde{\tau}^{\prime}(s)=\nu_{s}(\tilde{g}) .
$$

Furthermore, $s \mapsto \nu_{s}$ is weakly continuous 
since using Theorem 8.2 of [17] one easily shows that each weak limit point of a sequence $\left(\nu_{s_{k}}\right)_{k \geq 1}$ with $s_{k} \rightarrow s$ is an equilibrium state for $s \tilde{g}$ and hence is the unique equilibrium state $\nu_{s}$.

After this preparation fix $a \in H$. Set

$$
l(u)=\hat{\tau}(a)+a u \quad \text { for } u \in \mathbb{R} \quad \text { and } \quad L=\{s \in \mathbb{R}: \tau(s)-l(s)=0\} .
$$

By the definition of $H$ and $\hat{\tau}$ we have $l(u) \leq \tau(u)$ and $\inf _{u \in \mathbb{R}}(\tau(u)-l(u))=0$. Therefore $l$ is a tangent to $\tau$ with slope $a$. Since $\tau$ is convex, either $L$ is a closed interval, which may be unbounded, or $L$ is empty. We have $\hat{\tau}(a)=$ $\tau(s)-$ as for all $s \in L$.

Suppose first that $L$ is bounded and not empty. Then $L=[p, q]$ with $p \leq q$. In order to find $\mu_{k}$, fix $k \geq 1$ and let $G$ be a finite subset of $L$ containing $p$ and $q$ such that two adjacent points in $G$ have distance $<1 / k$. Set $v=p-1 / k$ and $w=q+1 / k$. Choose $\delta \in(0,1 / k)$ with $3 \delta<\tau(u)-l(u)$ for $u \in\{v, w\}$. Set $F=G \cup\{v, w\}$ and let $\tilde{g}$ and $B$ be as above such that 4.1 holds. By 4.3), 4.1 and since $\tilde{\tau}$ is convex with $\tau(p)=l(p)$ and $\tau(v)>l(v)+3 \delta$, we have

$\nu_{v}(\tilde{g})=\tilde{\tau}^{\prime}(v)<\frac{\tilde{\tau}(p)-\tilde{\tau}(v)}{1 / k}<\frac{\tau(p)-\tau(v)+2 \delta}{1 / k}<\frac{l(p)-l(v)-\delta}{1 / k}=a-k \delta$.

Similarly we get $\nu_{w}(\tilde{g})>a+k \delta$. By 4.1) we have $\nu_{v}(g) \leq \nu_{v}(\tilde{g})+\delta<a$ and $\nu_{w}(g) \geq \nu_{w}(\tilde{g})>a$. The measures $\nu_{s}$ assign measure zero to single points. Therefore $s \mapsto \nu_{s}(g)$ is continuous by (4.4), since the set where $g$ is discontinuous is at most countable. By the mean value theorem there is $z \in(v, w)$ with $\nu_{z}(g)=a$. By the choice of $G$ there is $y \in G$ with $|z-y|<1 / k$. We write $C$ for $\max (|p|,|q|)$, so that $|y| \leq C$. By (4.1) and (4.3),

$$
\begin{aligned}
& \left|z \nu_{z}(\tilde{g})-y \nu_{z}(g)\right| \leq\left|z \nu_{z}(\tilde{g})-y \nu_{z}(\tilde{g})\right|+\left|y \nu_{z}(\tilde{g})-y \nu_{z}(g)\right|<\frac{1}{k}\|g\|_{\infty}+C \delta, \\
& |\tilde{\tau}(z)-\tau(y)| \leq|\tilde{\tau}(z)-\tilde{\tau}(y)|+|\tilde{\tau}(y)-\tau(y)|<\frac{1}{k}\left\|\tilde{\tau}^{\prime}\right\|_{\infty}+\delta \leq \frac{1}{k}\|g\|_{\infty}+\delta .
\end{aligned}
$$

Applying these estimates to $h_{\nu_{z}}+z \nu_{z}(\tilde{g})-\tilde{\tau}(z)=0$, which follows from 4.2 and 4.3), we obtain

$$
\left|h_{\nu_{z}}+y \nu_{z}(g)-\tau(y)\right|<\frac{2}{k}\|g\|_{\infty}+\delta(1+C)<\frac{1}{k}\left(1+C+2\|g\|_{\infty}\right) .
$$

Furthermore, since $y \in G \subset L$ we have $\tau(y)-y \nu_{z}(g)=\tau(y)-y a=\hat{\tau}(a)$.

Set $\mu_{k}=\nu_{z}$. We have shown that $\mu_{k}$ is an ergodic invariant probability measure on $A$ satisfying $\mu_{k}(g)=a$ and $\left|h_{\mu_{k}}-\hat{\tau}(a)\right|<(1 / k)\left(1+C+2\|g\|_{\infty}\right)$, which implies $\lim _{k \rightarrow \infty} h_{\mu_{k}}=\hat{\tau}(a)$. Because $h_{\mu_{k}} \geq 0$ we also have $\hat{\tau}(a) \geq 0$. Hence the desired results are proved in the case where $L$ is bounded and nonempty. 
Now suppose that $L$ is empty or unbounded. Then $\lim _{s \rightarrow \infty}(\tau(s)-l(s))$ $=0$ or $\lim _{s \rightarrow-\infty}(\tau(s)-l(s))=0$. Let $\left(s_{k}\right)_{k \geq 1}$ be such that $\tau$ is differentiable at $s_{k}$ for $k \geq 1$ and $s_{k} \uparrow \infty$ in the first case, and $s_{k} \downarrow-\infty$ in the second. Set $a_{k}=\tau^{\prime}\left(s_{k}\right)$. Then $a_{k} \rightarrow a$ and $\hat{\tau}\left(a_{k}\right) \rightarrow \hat{\tau}(a)$ as $k \rightarrow \infty$, since $\tau$ is convex.

In order to find the measures $\mu_{k}$, fix $k \geq 1$ and choose $\varepsilon \in(0,1)$ such that

$$
\left|\frac{\tau\left(s_{k}+h\right)-\tau\left(s_{k}\right)}{h}-a_{k}\right|<\frac{1}{k\left|s_{k}\right|} \quad \text { for } h \in\{-\varepsilon, \varepsilon\} .
$$

Let $\tilde{g}$ and $B$ be as above such that (4.1) holds with $\delta=\varepsilon / k\left|s_{k}\right|$ and $F=$ $\left\{s_{k}-\varepsilon, s_{k}, s_{k}+\varepsilon\right\}$. Let $\tilde{\tau}$ and the measures $\nu_{s}$ be as above. Since $|\tau(s)-\tilde{\tau}(s)|$ $<\delta$ for all $s \in F$ by (4.1) we get

$$
\left|\frac{\tau\left(s_{k}+h\right)-\tau\left(s_{k}\right)}{h}-\frac{\tilde{\tau}\left(s_{k}+h\right)-\tilde{\tau}\left(s_{k}\right)}{h}\right|<\frac{2 \delta}{\varepsilon}=\frac{2}{k\left|s_{k}\right|}
$$

and hence also

$$
\left|\frac{\tilde{\tau}\left(s_{k}+h\right)-\tilde{\tau}\left(s_{k}\right)}{h}-a_{k}\right|<\frac{3}{k\left|s_{k}\right|} \quad \text { for } h \in\{-\varepsilon, \varepsilon\} .
$$

As $\tilde{\tau}$ is convex, $\tilde{\tau}^{\prime}\left(s_{k}\right)$ lies between $\frac{\tilde{\tau}\left(s_{k}+\varepsilon\right)-\tilde{\tau}\left(s_{k}\right)}{\varepsilon}$ and $\frac{\tilde{\tau}\left(s_{k}-\varepsilon\right)-\tilde{\tau}\left(s_{k}\right)}{-\varepsilon}$ and we get

$$
\left|\tilde{\tau}^{\prime}\left(s_{k}\right)-a_{k}\right|<\frac{3}{k\left|s_{k}\right|} .
$$

Set $\mu_{k}=\nu_{s_{k}}$ for $k \geq 1$. By (4.1) we get $\left|\mu_{k}(g)-\mu_{k}(\tilde{g})\right| \leq \delta$. By (4.3) we have $\tilde{\tau}^{\prime}\left(s_{k}\right)=\mu_{k}(\tilde{g})$, and so $\left|\mu_{k}(g)-a_{k}\right|<\delta+3 / k\left|s_{k}\right|<4 / k\left|s_{k}\right|$ from (4.5). This implies $\lim _{k \rightarrow \infty} \mu_{k}(g)=\lim _{k \rightarrow \infty} a_{k}=a$. Now, by (4.2) and (4.3) we have $h_{\mu_{k}}=\tilde{\tau}\left(s_{k}\right)-s_{k} \mu_{k}(\tilde{g})=\tilde{\tau}\left(s_{k}\right)-s_{k} \tilde{\tau}^{\prime}\left(s_{k}\right)$. Because $\hat{\tau}\left(a_{k}\right)=\tau\left(s_{k}\right)-a_{k} s_{k}$ we get $\left|h_{\mu_{k}}-\hat{\tau}\left(a_{k}\right)\right| \leq\left|\tilde{\tau}\left(s_{k}\right)-\tau\left(s_{k}\right)\right|+\left|s_{k}\right| \cdot\left|\tilde{\tau}^{\prime}\left(s_{k}\right)-a_{k}\right|<\delta+\frac{3}{k}<\frac{1}{k}\left(\frac{1}{\left|s_{k}\right|}+3\right)$ using 4.1) and 4.5. This implies $\lim _{k \rightarrow \infty} h_{\mu_{k}}=\lim _{k \rightarrow \infty} \hat{\tau}\left(a_{k}\right)=\hat{\tau}(a)$. Because $h_{\mu_{k}} \geq 0$ we also get $\hat{\tau}(a) \geq 0$.

Now we consider the level sets $L_{a}=\left\{x \in A: \lim _{n \rightarrow \infty} n^{-1} S_{n} g(x)=a\right\}$.

TheOREM 10. Let $g:[0,1] \rightarrow \mathbb{R}$ be a regular function and let $A$ be a completely invariant topologically transitive closed subset of $([0,1], T)$ with $h_{\text {top }}(T \mid A)>0$. For all $a \in H$ with $\hat{\tau}(a)>0$ there is an ergodic invariant probability measure $\mu$ on $A$ with $h_{\mu} \geq \hat{\tau}(a)$ and $\mu\left(L_{a}\right)=1$.

Proof. We consider the following compactification of the dynamical system $([0,1], T)$. Let $F \subset[0,1]$ be a finite or countable set such that $T$ and $g$ are continuous on $[0,1] \backslash F$. Set $W=\bigcup_{j=0}^{\infty} T^{-j} F$ and replace each $x \in W \backslash\{0,1\}$ by two points $x$ - and $x+$. We denote this extended interval $[0,1]$ by $X$; it is compact with respect to the order topology. The extensions of $T$ and $g$ from $[0,1] \backslash W$ to $X$ are continuous. Let $\bar{A}$ be the closure of $A \backslash W$ in $X$. 
Let $\mathcal{L}$ be the set of all $T$-invariant probability measures on $\bar{A}$ satisfying $\mu(g)=a$ and $h_{\mu} \geq \hat{\tau}(a)$. Let $\left(\mu_{k}\right)_{k \geq 1}$ be the sequence of probability measures found in Proposition 9. Because $\lim _{k \rightarrow \infty} h_{\mu_{k}}=\hat{\tau}(a)>0$, we have $h_{\mu_{k}}>0$ for all $k$ greater than or equal to some $k_{0}$. Since these measures are also ergodic, they assign measure zero to the countable set $W$. Therefore we can consider these measures $\mu_{k}$ as measures on $A \backslash W \subset X$. Let $\mu$ be a limit point of the sequence $\left(\mu_{k}\right)_{k \geq k_{0}}$ in $X$. Then $\mu$ is concentrated on $\bar{A}$ and satisfies $h_{\mu} \geq \hat{\tau}(a)$ and $\mu(g)=a$. This shows that $\mathcal{L}$ is not empty.

Moreover, $\mathcal{L}$ is a compact and convex subset of the set of all $T$-invariant probability measures on $\bar{A}$. Hence there is an ergodic probability measure $\mu$ in $\mathcal{L}$. The assumption $\hat{\tau}(a)>0$ implies $h_{\mu}>0$. Thus countable sets have $\mu$-measure zero and $\mu$ can again be considered as a measure on $A$, since $\bar{A} \backslash(A \backslash W)$ is at most countable. Hence we have found an ergodic probability measure $\mu$ on $A$ with $\mu(g)=a$ and $h_{\mu} \geq \hat{\tau}(a)$. By the ergodic theorem we have $\lim _{n \rightarrow \infty} n^{-1} S_{n} g(x)=\mu(g)=a$ for $\mu$-almost all $x \in A$, which implies $\mu\left(L_{a}\right)=1$.

5. Legendre transforms. For a topologically transitive completely invariant closed subset $A$ of $([0,1], T)$ with $h_{\text {top }}(T \mid A)>0$ and a regular function $g:[0,1] \rightarrow \mathbb{R}$ we introduced the pressure function $\tau(s)=q(T \mid A, s g)$ for $s \in \mathbb{R}$ and its Legendre transform $\hat{\tau}$ defined by $\hat{\tau}(a)=\inf _{s \in \mathbb{R}}(\tau(s)-a s)$ on the set $H$ of all $a$ for which this infimum exists. Since $\tau$ is convex, $H$ is a nonempty interval.

We also introduce the function

$$
\check{\tau}(a)=\frac{1}{a} \hat{\tau}(a)=\frac{1}{a} \inf _{s \in \mathbb{R}}(\tau(s)-a s) \quad \text { for all } a \in H \backslash\{0\} .
$$

We shall use the Legendre transform $\hat{\tau}$ to characterize the entropy spectrum of Birkhoff averages, and the function $\check{\tau}$ to characterize the dimension spectrum of Lyapunov exponents. First we show some properties of the Legendre transform.

Proposition 11. Let $A$ be a completely invariant closed subset of $([0,1], T)$ with $h_{\mathrm{top}}(T \mid A)>0$ and let $g:[0,1] \rightarrow \mathbb{R}$ be a regular function. Let the pressure function $\tau$ and the transforms $\hat{\tau}$ and $\check{\tau}$, which are defined on the set $H$, be as above. Then $H$ equals $\{a \in \mathbb{R}:$ as $\leq \tau(s)$ for all $s \in \mathbb{R}\}$, which is a bounded closed interval, and $\hat{\tau}: H \rightarrow \mathbb{R}$ is concave and hence continuous and attains its supremum $\tau(0)$ on $H$. If there is $s_{0}>0$ with $\tau(s) \leq 0$ for $s \leq-s_{0}$ and $\tau(s)>0$ for $s>-s_{0}$, then $H$ is a subset of $[0, \infty)$, and $a \mapsto \check{\tau}(1 / a)$ can be extended to a concave and continuous function on $H^{-1}:=\{1 / a: a \in H\}$, which is a subinterval of $(0, \infty]$. Furthermore, $a \mapsto \check{\tau}(1 / a)$ attains its supremum $s_{0}$ on $H^{-1}$. 
Proof. If $a \in H$, then $\hat{\tau}(a)+a s \leq \tau(s)$ for all $s \in \mathbb{R}$ by the definition of $\hat{\tau}$, and Proposition 9 says that $\hat{\tau}(a) \geq 0$. Therefore $a s \leq \tau(s)$ for all $s \in \mathbb{R}$. This shows $H \subset\{a \in \mathbb{R}: a s \leq \tau(s)$ for all $s \in \mathbb{R}\}$. Since as $\leq \tau(s)$ for all $s \in \mathbb{R}$ implies that $\inf _{s \in \mathbb{R}}(\tau(s)-a s)$ exists, we also get $H \supset\{a \in \mathbb{R}: a s \leq$ $\tau(s)$ for all $s \in \mathbb{R}\}$.

One sees that $H=\{a \in \mathbb{R}: a s \leq \tau(s)$ for all $s \in \mathbb{R}\}$ is a closed interval. If $a \in H$ we have $a \leq \tau(1)$ and $-a \leq \tau(-1)$. This gives $H \subset[-\tau(-1), \tau(1)]$.

By its definition, $\hat{\tau}$ is the infimum of linear functions and hence is concave. Since $\tau$ is convex, there is $a \in \mathbb{R}$ such that $\tau(s) \geq \tau(0)+a s$ for all $s \in \mathbb{R}$. The definition of $\hat{\tau}$ implies that $\hat{\tau}(a) \geq \tau(0)$ for this $a$. Furthermore, $\hat{\tau}(a)=\inf _{s \in \mathbb{R}}(\tau(s)-a s) \leq \tau(0)$ for all $a \in H$. Hence $\hat{\tau}$ attains its supremum $\tau(0)$ in $H$.

Now suppose that there is $s_{0}>0$ with $\tau(s) \leq 0$ for $s \leq-s_{0}$ and $\tau(s)>0$ for $s>-s_{0}$. If $a \in H$, then $-a s_{0} \leq \tau\left(-s_{0}\right) \leq 0$, which implies $a \geq 0$. This shows that $H \subset[0, \infty)$. For $a \in H \backslash\{0\}$ we have $\check{\tau}(a)=$ $(1 / a) \hat{\tau}(a) \geq 0$ by Proposition 9 and $\check{\tau}(a)=(1 / a) \hat{\tau}(a) \leq(1 / a) \tau\left(-s_{0}\right)+s_{0}$ $\leq s_{0}$ by definition of $\hat{\tau}$. This implies that $a \mapsto \check{\tau}(1 / a)$ is a bounded function on $H^{-1} \backslash\{\infty\}$, which is concave and continuous, since it is the infimum of the linear functions $a \mapsto a \tau(s)-s$ over $s \in \mathbb{R}$. Therefore we can extend $a \mapsto \check{\tau}(1 / a)$ to a continuous concave function on $H^{-1}$, which is bounded below by 0 and above by $s_{0}$.

By the convexity of $\tau$ and the properties of $s_{0}$, for every $n \geq 1$ there is $b_{n}>0$ such that $\tau(s) \geq b_{n}\left(s+s_{0}-1 / n\right)$ for all $s \in \mathbb{R}$, which implies $\hat{\tau}\left(b_{n}\right) \geq b_{n}\left(s_{0}-1 / n\right)$ and $\check{\tau}\left(b_{n}\right) \geq s_{0}-1 / n$. Since $H$ is a compact interval, the sequence $\left(b_{n}\right)_{n \geq 1}$ has a limit point $b$ in $H$. By the above results, $\check{\tau}$ has a continuous extension to $H$, and we get $\check{\tau}(b) \geq s_{0}$. This shows that $a \mapsto$ $\check{\tau}(1 / a)$ attains its supremum $s_{0}$ in $H^{-1}$.

It may happen that the function $\check{\tau}: H \rightarrow \mathbb{R}$ is not concave. The following example is similar to an example given in [2]. Choose $u \in(0, \log 2)$ and set $v=-\log \left(1-e^{-u}\right)$. We have $0<u<v$. Let $T:[0,1] \rightarrow[0,1]$ be defined by

$$
T(x)= \begin{cases}e^{u} x & \text { for } 0 \leq x \leq e^{-u}, \\ -e^{v}(x-1) & \text { for } e^{-u} \leq x \leq 1 .\end{cases}
$$

The set $A=[0,1]$ is completely invariant and topologically transitive. Let $g:[0,1] \rightarrow \mathbb{R}$ be defined by $g(x)=u$ for $1 \leq x<e^{-u}$ and $g(x)=v$ for $e^{-u}<x \leq 1$, so $g=\log \left|T^{\prime}\right|$. For $g\left(e^{-u}\right)$ we can choose any value in $[u, v]$. We have $\tau(s)=p(T \mid A, s g)=\log \left(e^{s u}+e^{s v}\right)$ since $A$ itself is a Markov subset. The function $\tau: \mathbb{R} \rightarrow \mathbb{R}$ is convex and differentiable and the set of values of its derivative is the interval $(u, v)$. This implies that $H=[u, v]$. Computing the infimum in the definition of $\hat{\tau}$, one gets 


$$
\hat{\tau}(a)=\log \left(\left(\frac{v-a}{a-u}\right)^{\frac{a-u}{v-u}}+\left(\frac{a-u}{v-a}\right)^{\frac{v-a}{v-u}}\right) \quad \text { for } a \in H .
$$

If one draws the graph of the function $a \mapsto(1 / a) \hat{\tau}(a)=\check{\tau}(a)$ for $u=0.04$, then it turns out that this function is not concave.

This example also shows that the interval $H$ need not contain the point zero. To get an example for which $0 \in H$, let $T:[0,1] \rightarrow[0,1]$ be defined by

$$
T(x)= \begin{cases}\frac{x}{1-x} & \text { for } 0 \leq x \leq 1 / 2 \\ -2(x-1) & \text { for } 1 / 2 \leq x \leq 1\end{cases}
$$

Again, the set $A=[0,1]$ is completely invariant and topologically transitive. For $g$ we choose again the map $\varphi=-\log \left|T^{\prime}\right|$, which has value 0 at the fixed point 0 and satisfies $\varphi(x)>0$ for $x \in(0,1]$. It follows that $\tau(s)=p(T \mid A, s g)$ satisfies $\tau(s) \geq s g(0)=0$ for all $s \in \mathbb{R}$. By Lemma 12 below there is $s_{0}>0$ such that $\tau(s)=0$ for $s \leq-s_{0}$. This implies that 0 is the left endpoint of $H$, and we have an example with $0 \in H$. In this case $\infty \in H^{-1}$ and by Proposition 11 the function $a \mapsto \check{\tau}(1 / a)$ must attain its supremum at $\infty$ and therefore $\check{\tau}: H \rightarrow \mathbb{R}$ attains its supremum at 0 .

Now we consider the case where $g$ is the function $\log \left|T^{\prime}\right|$.

LEMMA 12. Let A be a topologically transitive completely invariant closed subset of $([0,1], T)$ with $h_{\mathrm{top}}(T \mid A)>0$. Suppose that $\varphi=\log \left|T^{\prime}\right|$ is a regular function and set $\tau(s)=q(T \mid A, s \varphi)$ for $s \in \mathbb{R}$. Then $\tau: \mathbb{R} \rightarrow \mathbb{R}$ is a nondecreasing continuous function. Furthermore, there is $s_{0} \in(0,1]$ with $\tau(s) \leq 0$ for $s \leq-s_{0}$ and $\tau(s)>0$ for $s>-s_{0}$.

Proof. Let $\mathcal{Z}$ be a partition which is a generator and with respect to which $T$ is piecewise monotone. From Lemma 8 we deduce that $\tau(s)=$ $\sup _{\mu \in \mathcal{E}(A, \mathcal{Z})}\left(h_{\mu}+s \mu(\varphi)\right)$. In particular, $\tau$ is a convex continuous function. We show that $\mu(\varphi) \geq 0$ for all $\mu \in \mathcal{E}(A, \mathcal{Z})$.

To this end suppose that there is a probability measure $\mu \in \mathcal{E}(A, \mathcal{Z})$ with $\mu(\varphi)<0$. Since $\mu$ assigns measure zero to single points, we find a continuous function $f:[0,1] \rightarrow \mathbb{R}$ satisfying $f \geq \varphi$ and $\mu(f)<0$. Since $\mu$ is supported on a Markov subset $B$ and Markov subsets have the specification property, there is a probability measure $\pi$ which is concentrated on a periodic orbit and satisfies $\pi(f)<0$. Because $f \geq \varphi$ we get $\pi(\varphi)<0$. This means that we have an attracting periodic orbit, contradicting the fact that $\mathcal{Z}$ is a generator. Therefore $\mu(\varphi) \geq 0$ for all $\mu \in \mathcal{E}(A, \mathcal{Z})$.

Now $\tau$ is the supremum of linear functions of nonnegative slope each. This implies that $\tau$ is nondecreasing. Furthermore, for $\mu \in \mathcal{E}(A, \mathcal{Z})$ we have $h_{\mu} \leq \mu(\varphi)$. If $h_{\mu}=0$, this follows from $\mu(\varphi) \geq 0$. If $h_{\mu}>0$, it follows from Theorem 2 in [6]. Because $h_{\mu}-\mu(\varphi) \leq 0$ for all $\mu \in \mathcal{E}(A, \mathcal{Z})$ we get $\tau(-1) \leq 0$. By Lemma 7 we have $\tau(0)=q(T \mid A, 0)=h_{\text {top }}(T \mid A)>0$. Since 
$\tau$ is nondecreasing and continuous, there is an $s_{0}$ with the desired properties.

6. Exceptional sets. In order to get upper estimates of entropy and Hausdorff dimension, we have to consider certain exceptional sets. Let $\mathcal{Z}$ be a partition of $[0,1]$ with respect to which $T$ is piecewise monotone. Set $R_{\mathcal{Z}}=$ $\bigcap_{i=0}^{\infty} T^{-i}\left(\bigcup_{Z \in \mathcal{Z}} Z\right)$. For $n \geq 1$ let $Z_{n}(x)$ be the element of $\mathcal{Z}_{n}=\bigvee_{j=0}^{n-1} T^{-j} \mathcal{Z}$ which contains $x$. If $x \in R_{\mathcal{Z}}$ then $Z_{n}(x)$ is defined for all $n \geq 1$.

Let $A$ be a completely invariant closed subset of $([0,1], T)$ and let $\varrho$ be a probability measure on $A$. For an open interval $(p, q)$ and $r \in(p, q)$ set

$$
\varrho_{r}((p, q))=\min \{\varrho((p, r)), \varrho((r, s))\} .
$$

This can be considered as a distance of the point $r$ to the boundary of the interval $(p, q)$ where the distance is determined by the measure $\varrho$. For $x \in R_{\mathcal{Z}}$ and $d>0$ set

$$
I_{d}(x)=\left\{n \geq 1: \varrho_{T^{n}(x)}\left(T^{n} Z_{n}(x)\right) \geq d\right\}
$$

and $N_{d}=N_{0} \cup\left\{x \in A \cap R_{\mathcal{Z}}: \operatorname{card} I_{d}(x)<\infty\right\}$ where $N_{0}=A \backslash R_{\mathcal{Z}}$.

In order to show that the sets $N_{d}$ are small, for a partition $\mathcal{Z}$ of the interval $[0,1]$ and a function $f:[0,1] \rightarrow \mathbb{R}$ we define

$$
\operatorname{var}_{\mathcal{Z}}(f)=\sup _{Z \in \mathcal{Z}} \sup _{x, y \in Z}|f(x)-f(y)|
$$

and prove the following lemma.

Lemma 13. Let $\mathcal{Z}$ be a partition of $[0,1]$ with respect to which $T$ is piecewise monotone, and set $\varphi=\log \left|T^{\prime}\right|$. Suppose that $\operatorname{var}_{\mathcal{Z}}(\varphi)<\alpha$. Then for every $x \in R_{\mathcal{Z}}$ with $\underline{\chi}(x) \geq \alpha$ there is $c_{x}$ such that $\left|Z_{n}(x)\right| \leq e^{-n \gamma}$ for all $n \geq c_{x}$, where $\gamma=\frac{1}{2}\left(\alpha-\operatorname{var}_{\mathcal{Z}}(\varphi)\right)$.

Proof. Because $\chi(x) \geq \alpha$ there is $c_{x}$ such that $S_{n} \varphi(x) \geq n(\alpha-\gamma)$ for all $n \geq c_{x}$. For all $y \in Z_{n}(x)$ we have $S_{n} \varphi(y) \geq S_{n} \varphi(x)-n \operatorname{var}_{\mathcal{Z}}(\varphi)$, which gives $\left|Z_{n}(x)\right| \leq\left|T^{n}\left(Z_{n}(x)\right)\right| \sup _{Z_{n}(x)} e^{-S_{n} \varphi} \leq e^{-S_{n} \varphi(x)+n \operatorname{var}_{\mathcal{Z}}(\varphi)} \leq e^{-n \gamma}$ for all $n \geq c_{x}$.

Lemma 14. Let $A$ be a completely invariant closed subset of $([0,1], T)$ and let $\varrho$ be a probability measure with support $A$. For a partition $\mathcal{Z}$ of $[0,1]$ with respect to which $T$ is piecewise monotone, let $N_{d}$ be the set defined above. Then $\lim _{d \downarrow 0} \operatorname{ent}_{\mathrm{B}}\left(N_{d}\right)=0$. Furthermore, for every $\alpha>0$ we have $\lim _{d \downarrow 0} \operatorname{dim}_{\mathrm{H}}\left(N_{d} \cap D_{\alpha}\right)=0$ if $\operatorname{var}_{\mathcal{Z}}(\varphi)<\alpha$, where $D_{\alpha}=\{x \in A: \underline{\chi}(x) \geq \alpha\}$ and $\varphi=\log \left|T^{\prime}\right|$.

Proof. For $l \geq 1$ and $Z \in \mathcal{Z}_{l}$ we define

$$
G_{l, Z}=\left\{x \in Z \cap A \cap R_{\mathcal{Z}}: \varrho_{T^{n}(x)}\left(T^{n} Z_{n}(x)\right)<d \text { for all } n \geq l\right\} .
$$


We estimate the entropy of these sets. For fixed $d>0$ small enough choose $k=k(d)$ maximal such that $\varrho(Y) \geq d$ for all $Y \in \mathcal{Z}_{k}$ with $Y \cap A \neq \emptyset$. Since $\varrho$ has support $A$, all open intervals $Y$ with $Y \cap A \neq \emptyset$ satisfy $\varrho(Y)>0$. This implies $\lim _{d \downarrow 0} k(d)=\infty$.

For every $n \geq 0$ we construct a subset $\mathcal{C}_{n}$ of $\mathcal{Z}_{l+n k}$ which satisfies card $\mathcal{C}_{n} \leq 4^{n}$ and $G_{l, Z} \subset \bigcup_{C \in \mathcal{C}_{n}} C$. We start with $\mathcal{C}_{0}=\{Z\}$. Suppose that $\mathcal{C}_{j}$ is constructed. For a set $Y \in \mathcal{C}_{j} \subset \mathcal{Z}_{l+j k}$ we have $\varrho_{T^{l+j k}(x)}\left(T^{l+j k} Y\right)<d$ for all $x \in Y \cap G_{l, Z}$. Therefore $T^{l+j k}\left(Y \cap G_{l, Z}\right)$ is contained in the closure of two open intervals with $\varrho$-measure at most $d$ each and a common endpoint with the interval $T^{l+j k} Y$. By the choice of $k=k(d)$ there is a subset $\mathcal{A}_{Y}$ of $\mathcal{Z}_{k}$ with card $\mathcal{A}_{Y} \leq 4$ such that $T^{l+j k}\left(Y \cap G_{l, Z}\right) \subset \bigcup_{E \in \mathcal{A}_{Y}} \bar{E}$. Set $\mathcal{B}_{Y}=\left\{Y \cap T^{-l-j k}(E): E \in \mathcal{A}_{Y}\right\}$. We have $Y \cap G_{l, Z} \subset \bigcup_{C \in \mathcal{B}_{Y}} \bar{C}$, which implies $Y \cap G_{l, Z} \subset \bigcup_{C \in \mathcal{B}_{Y}} C$, since the endpoints of the intervals in $\mathcal{B}_{Y}$ are not in $R_{\mathcal{Z}}$. Set $\mathcal{C}_{j+1}=\bigcup_{Y \in \mathcal{C}_{j}} \mathcal{B}_{Y}$. This is a subset of $\mathcal{Z}_{l+(j+1) k}$ of cardinality less than or equal to $4^{j+1}$, which satisfies $G_{l, Z} \subset \bigcup_{C \in \mathcal{C}_{j+1}} C$. This finishes the construction of the sequence $\left(\mathcal{C}_{n}\right)_{n \geq 0}$.

We estimate $\operatorname{ent}_{\mathrm{B}}\left(N_{d}\right)$. For $t=2 / k$ and $n \geq 1$ we have

$$
\sum_{C \in \mathcal{C}_{n}} e^{-t \ell(C)} \leq 4^{n} e^{-t(l+n k)}=e^{-t l}\left(4 / e^{2}\right)^{n} .
$$

This implies $\gamma_{t}\left(G_{l, Z}\right)=0$. As $N_{d} \subset N_{0} \cup \bigcup_{l=1}^{\infty} \bigcup_{Z \in \mathcal{Z}_{l}} G_{l, Z}$ and as $N_{0}$ is countable, we get $\gamma_{t}\left(N_{d}\right)=0$. Therefore $\operatorname{ent}_{\mathrm{B}}\left(N_{d}\right) \leq t=2 / k$. Since $k$ tends to infinity as $d$ goes to zero, this implies $\lim _{d \downarrow 0} \operatorname{ent}_{\mathrm{B}}\left(N_{d}\right)=0$.

Now we estimate the Hausdorff dimension of $N_{d} \cap D_{\alpha}$. To this end set $\gamma=\frac{1}{2}\left(\alpha-\operatorname{var}_{\mathcal{Z}}(\varphi)\right)$, and for $x \in D_{\alpha} \cap R_{\mathcal{Z}}$ let $c_{x}$ be as in Lemma 13 . Set $D_{m}=\left\{x \in D_{\alpha} \cap R_{\mathcal{Z}}: c_{x} \leq m\right\}$ and $\mathcal{D}_{n}=\left\{C \in \mathcal{C}_{n}: C \cap D_{m} \neq \emptyset\right\}$ for $m, n \geq 1$. By Lemma 13 we have $|C| \leq e^{-(l+n k) \gamma}$ for all $C \in \mathcal{D}_{n}$, if $n \geq m$. Setting $t=2 / \gamma k$ we get, for $n \geq m$,

$$
\sum_{C \in \mathcal{D}_{n}}|C|^{t} \leq 4^{n} e^{-t(l+n k) \gamma}=e^{-t l \gamma}\left(4 / e^{2}\right)^{n} .
$$

Because $G_{l, Z} \cap D_{m} \subset \bigcup_{C \in \mathcal{D}_{n}} C$, we get $\nu_{t}\left(G_{l, Z} \cap D_{m}\right)=0$. By Lemma 13 we have $\bigcup_{m=1}^{\infty} D_{m}=D_{\alpha} \cap R_{\mathcal{Z}}$. Therefore $\nu_{t}\left(G_{l, Z} \cap D_{\alpha}\right)=0$. As $N_{d} \subset$ $N_{0} \cup \bigcup_{l=1}^{\infty} \bigcup_{Z \in \mathcal{Z}_{l}} G_{l, Z}$ and as $N_{0}$ is countable, we get $\nu_{t}\left(N_{d} \cap D_{\alpha}\right)=0$. This gives $\operatorname{dim}_{\mathrm{H}}\left(N_{d} \cap D_{\alpha}\right) \leq t=2 / \gamma k$. Since $k$ tends to infinity as $d$ goes to zero, we conclude that $\lim _{d \downarrow 0} \operatorname{dim}_{\mathrm{H}}\left(N_{d} \cap D_{\alpha}\right)=0$.

We finish with a lemma, which we shall need in Section 8 .

Lemma 15. Let $\varrho$ be a probability measure on $[0,1]$. For every $d>0$ there is $b>0$ such that for any open interval $I \subset[0,1]$ and any $u \in I$ with $\varrho_{u}(I) \geq d$ we have $|I| \geq b$. 
Proof. Fix $d>0$. Suppose that for every $n \geq 1$ there are an open interval $I_{n}$ and a point $u_{n} \in I_{n}$ with $\varrho_{u_{n}}\left(I_{n}\right) \geq d$ such that $\lim _{n \rightarrow \infty}\left|I_{n}\right|=0$. Let $u$ be a limit point of $\left(u_{n}\right)_{n \geq 1}$. Because $\varrho_{u_{n}}\left(I_{n}\right) \geq d$ for all $n \geq 1$, $u$ cannot be an endpoint of $[0,1]$. Choose $v>0$ such that $\varrho([u-2 v, u))<d$ and $\varrho((u, u+2 v])<d$. For infinitely many $n$ we have either $[u-2 v, u-v] \subset I_{n}$ or $[u+v, u+2 v] \subset I_{n}$, contradicting $\lim _{n \rightarrow \infty}\left|I_{n}\right|=0$.

7. The entropy spectrum of Birkhoff averages. Let $g:[0,1] \rightarrow \mathbb{R}$ be a regular function and let $A$ be a completely invariant closed subset of $([0,1], T)$. In order to give the definition of the sets whose entropy we shall estimate, we split $H$ into intervals on which $\hat{\tau}$ is constant, increasing and decreasing. Set $H_{0}=\{a \in H: \hat{\tau}(a)=\tau(0)\}$. This is the set where $\hat{\tau}$ attains its supremum. Since $\hat{\tau}$ is concave, $H_{0}$ is a closed interval, possibly a single point. Set $H_{+}=\left\{a \in H: a>b\right.$ for all $\left.b \in H_{0}\right\}$ and $H_{-}=\{a \in H: a<b$ for all $\left.b \in H_{0}\right\}$. For $a \in H$ we define

$$
U_{a}= \begin{cases}\left\{x \in A: \limsup _{n \rightarrow \infty} n^{-1} S_{n} g(x) \leq a\right\} & \text { if } a \in H_{-}, \\ A & \text { if } a \in H_{0}, \\ \left\{x \in A: \liminf _{n \rightarrow \infty} n^{-1} S_{n} g(x) \geq a\right\} & \text { if } a \in H_{+} .\end{cases}
$$

Now we can estimate the entropy of these sets.

TheOREM 16. Let $g:[0,1] \rightarrow \mathbb{R}$ be a regular function and let $A$ be a completely invariant topologically transitive closed subset of $([0,1], T)$ with $h_{\text {top }}(T \mid A)>0$. For each $a \in H$ we have $\operatorname{ent}_{\mathrm{B}}\left(U_{a}\right) \leq \hat{\tau}(a)$.

Proof. For each $a \in H_{0}$ we have $\hat{\tau}(a)=\tau(0)$. Since $\tau$ is a convex function, the definition of $\hat{\tau}$ implies that $\hat{\tau}(a)=\inf _{s<0}(\tau(s)-a s)$ if $a \in H_{-}$, and $\hat{\tau}(a)=\inf _{s>0}(\tau(s)-a s)$ if $a \in H_{+}$.

Fix $a \in H$ and $\varepsilon>0$. Choose $s \in \mathbb{R}$ such that $\tau(s)-s a<\hat{\tau}(a)+\varepsilon$. We can do this in such a way that $s=0$ if $a \in H_{0}, s<0$ if $a \in H_{-}$, and $s>0$ if $a \in H_{+}$.

Choose $\delta>0$ such that $\delta|s| \leq \varepsilon$. Let $\mathcal{Z}$ be a partition of $[0,1]$ with respect to which $T$ is piecewise monotone, and $\operatorname{such}$ that $\operatorname{var}_{\mathcal{Z}}(g)<\delta$, which means $\sup _{x, y \in Z}|g(x)-g(y)|<\delta$ for all $Z \in \mathcal{Z}$. This is possible, since $g$ is regular. There is a function $\tilde{g}$ which is constant on each interval in $\mathcal{Z}$ and such that $|\tilde{g}-g| \leq \delta$ and $q(T \mid A, s \tilde{g})=q(T \mid A, s g)=\tau(s)$. This is possible by the properties of the pressure function given in Lemma 6 .

Lemma 8 shows that the notion of pressure we use here is the same as in [9]. Hence we can apply Theorem 2 of [9], which together with Lemma 4 of [7] shows the existence of a probability measure $\varrho$ with support $A$ which is $e^{\tau(s)-s \tilde{g}_{\text {-conformal. This means that }} \varrho(T Y)}=\int_{Y} e^{\tau(s)-s \tilde{g}} d \varrho$ for all intervals $Y$ contained in elements of $\mathcal{Z}$. 
We choose $d>0$ such that $\operatorname{ent}_{\mathrm{B}}\left(N_{d}\right)<\varepsilon$, which is possible by Lemma 14 and set $W=\left\{x \in A \backslash N_{d}: \liminf _{n \rightarrow \infty} s n^{-1} S_{n} g(x) \geq s a\right\}$. We then have $U_{a} \subset W \cup N_{d}$ for all $a \in H$. We now estimate the entropy of the set $W$.

Fix $k \in \mathbb{N}$. The definition of $W$ implies $s S_{n} g(x) \geq n s a-n \varepsilon$ for all $x \in W$ and all but finitely many $n$. For each $x \in W$ choose $n_{x}$ such that

$$
n_{x} \in I_{d}(x), \quad n_{x} \geq k, \quad s S_{n_{x}} g(x) \geq n_{x} s a-n_{x} \varepsilon .
$$

Set $Y_{x}=Z_{n_{x}}(x)$, which is well defined because $W \subset R_{\mathcal{Z}}$. Since $\tilde{g}$ is constant on each element of $\mathcal{Z}$, we have $\varrho\left(T^{j+1} Y_{x}\right)=e^{\tau(s)-s \tilde{g}\left(T^{j}(x)\right)} \varrho\left(T^{j} Y_{x}\right)$ for $0 \leq$ $j \leq n_{x}-1$ by the definition of a conformal measure, which implies

$$
\varrho\left(Y_{x}\right)=e^{s S_{n_{x}} \tilde{g}(x)-n_{x} \tau(s)} \varrho\left(T^{n_{x}} Y_{x}\right) .
$$

By the definition of $\tilde{g}$ and the choice of $n_{x}$ and $\delta$ we get

$$
s S_{n_{x}} \tilde{g}(x) \geq s S_{n_{x}} g(x)-n_{x}|s| \delta \geq n_{x} s a-n_{x} \varepsilon-n_{x}|s| \delta \geq n_{x} s a-2 n_{x} \varepsilon .
$$

Because $n_{x} \in I_{d}(x)$ we have

$$
\varrho\left(T^{n_{x}} Y_{x}\right) \geq \varrho_{T^{n_{x}(x)}}\left(T^{n_{x}} Y_{x}\right) \geq d .
$$

Putting all this together we get

$$
\varrho\left(Y_{x}\right) \geq d e^{n_{x} s a-n_{x} \tau(s)-2 n_{x} \varepsilon} \quad \text { for all } x \in W .
$$

Set $\tilde{\mathcal{U}}=\left\{Y_{x}: x \in W\right\} \subset \bigcup_{j=k}^{\infty} \mathcal{Z}_{j}$. Since two elements of $\bigcup_{j=k}^{\infty} \mathcal{Z}_{j}$ are either disjoint or one contains the other, there is a subset $\mathcal{U}$ of $\tilde{\mathcal{U}}$ which still covers $W$ and consists of pairwise disjoint intervals. We write $n(Y)$ for $n_{x}$ if $Y=Y_{x}$. The above estimate implies

$$
\sum_{Y \in \mathcal{U}} e^{-(\tau(s)-s a+2 \varepsilon) n(Y)} \leq \frac{1}{d} \sum_{Y \in \mathcal{U}} \varrho(Y) \leq \frac{1}{d} .
$$

We have chosen $s \in \mathbb{R}$ such that $\tau(s)-s a<\hat{\tau}(a)+\varepsilon$, so we get

$$
\sum_{Y \in \mathcal{U}} e^{-(\hat{\tau}(a)+3 \varepsilon) n(Y)} \leq \frac{1}{d}
$$

Furthermore, $\ell(Y) \geq n(Y)$ because $Y \in \mathcal{Z}_{n(Y)}$. This gives

$$
\sum_{Y \in \mathcal{U}} e^{-(\hat{\tau}(a)+3 \varepsilon) \ell(Y)} \leq \frac{1}{d}
$$

as $\hat{\tau}(a)$ is nonnegative. Since $\mathcal{U} \subset \bigcup_{j=k}^{\infty} \mathcal{Z}_{j}$ and $k \in \mathbb{N}$ can be chosen arbitrary, we get $\gamma_{\hat{\tau}(a)+3 \varepsilon}(W) \leq 1 / d$. This implies $\operatorname{ent}_{\mathrm{B}}(W) \leq \hat{\tau}(a)+3 \varepsilon$. Furthermore, we have chosen $d$ so that $\operatorname{ent}_{\mathrm{B}}\left(N_{d}\right)<\varepsilon$ and $W$ so that $U_{a} \subset W \cup N_{d}$. This gives $\operatorname{ent}_{\mathrm{B}}\left(U_{a}\right) \leq \hat{\tau}(a)+3 \varepsilon$. Since $\varepsilon>0$ was arbitrary, we conclude that $\operatorname{ent}_{\mathrm{B}}\left(U_{a}\right) \leq \hat{\tau}(a)$. 
For $u, v \in \mathbb{R}$ with $u \leq v$ we now consider the set

$$
L_{u, v}=\left\{x \in A: \liminf _{n \rightarrow \infty} \frac{1}{n} S_{n} g(x) \geq u, \limsup _{n \rightarrow \infty} \frac{1}{n} S_{n} g(x) \leq v\right\} .
$$

The level set $L_{a}=\left\{x \in A: \lim _{n \rightarrow \infty} n^{-1} S_{n} g(x)=a\right\}$ is then the set $L_{a, a}$. We compute the entropy of these sets.

TheOREM 17. Let $g:[0,1] \rightarrow \mathbb{R}$ be a regular function and let $A$ be a completely invariant topologically transitive closed subset of $([0,1], T)$ with $h_{\mathrm{top}}(T \mid A)>0$. Suppose that $u$ and $v$ are in $\mathbb{R}$ with $u \leq v$ and $H \cap[u, v] \neq \emptyset$. Then $\operatorname{ent}_{\mathrm{B}}\left(L_{u, v}\right)=\max _{a \in H \cap[u, v]} \hat{\tau}(a)$.

Proof. By Theorem 10, for each $a \in H \cap[u, v]$ with $\hat{\tau}(a)>0$ there exists an ergodic invariant probability measure $\mu$ on $A$ with $\mu\left(L_{a}\right)=1$ and $h_{\mu} \geq$ $\hat{\tau}(a)$. By Lemma 4 we get $\operatorname{ent}_{\mathrm{B}}\left(L_{a}\right) \geq h_{\mu}$, which $\operatorname{implies} \operatorname{ent}_{\mathrm{B}}\left(L_{u, v}\right) \geq \hat{\tau}(a)$ because $L_{a} \subset L_{u, v}$. Therefore $\operatorname{ent}_{\mathrm{B}}\left(L_{u, v}\right) \geq \max _{a \in H \cap[u, v]} \hat{\tau}(a)$.

In order to show the opposite inequality, we divide $H$ into the sets $H_{-}$, $H_{+}$and $H_{0}$, which have been introduced above. If $H_{0} \cap[u, v] \neq \emptyset$, then $L_{u, v} \subset A=U_{b}$ for some $b \in H_{0}$, which implies

$$
\operatorname{ent}_{\mathrm{B}}\left(L_{u, v}\right) \leq \operatorname{ent}_{\mathrm{B}}\left(U_{b}\right) \leq \hat{\tau}(b)=\tau(0)=\max _{a \in H \cap[u, v]} \hat{\tau}(a)
$$

using Theorem 16. If $H_{0} \cap[u, v]=\emptyset$, then either $u \in H_{+}$or $v \in H_{-}$. In the first case we get $L_{u, v} \subset U_{u}$, which implies

$$
\operatorname{ent}_{\mathrm{B}}\left(L_{u, v}\right) \leq \operatorname{ent}_{\mathrm{B}}\left(U_{u}\right) \leq \hat{\tau}(u) \leq \max _{a \in H \cap[u, v]} \hat{\tau}(a)
$$

using Theorem 16. In the second case we get $L_{u, v} \subset U_{v}$, which implies

$$
\operatorname{ent}_{\mathrm{B}}\left(L_{u, v}\right) \leq \operatorname{ent}_{\mathrm{B}}\left(U_{v}\right) \leq \hat{\tau}(v) \leq \max _{a \in H \cap[u, v]} \hat{\tau}(a)
$$

again using Theorem 16 .

Proposition 11 shows that the set $H$ on which $\hat{\tau}$ is defined is a bounded closed interval. We denote its endpoints by $\min H$ and $\max H$. Let $K_{-}$be the set of all $x \in A$ with $\limsup _{n \rightarrow \infty} n^{-1} S_{n} g(x)<\min H$, and $K_{+}$be the set of all $x \in A$ with $\liminf _{n \rightarrow \infty} n^{-1} S_{n} g(x)>\max H$. Let $K$ be the union of these two sets. Then we have

TheOREM 18. Let $g:[0,1] \rightarrow \mathbb{R}$ be a regular function and let $A$ be a completely invariant topologically transitive closed subset of $([0,1], T)$ with $h_{\text {top }}(T \mid A)>0$. Then $\operatorname{ent}_{\mathrm{B}}(K)=0$.

Proof. Suppose that ent $\mathrm{B}_{\mathrm{B}}(K)>0$. Then either $\operatorname{ent}_{\mathrm{B}}\left(K_{+}\right)>0 \operatorname{or~ent}_{\mathrm{B}}\left(K_{-}\right)$ $>0$. We give the proof for the first case; the other case is similar.

For $c \in \mathbb{R}$ set $K_{c}=\left\{x \in A: \liminf _{n \rightarrow \infty} n^{-1} S_{n} g(x) \geq c\right\}$. Since $\operatorname{ent}_{\mathrm{B}}\left(K_{+}\right)>0$, there exists $c>\max H$ satisfying $\operatorname{ent}_{\mathrm{B}}\left(K_{c}\right)>0$. Set $\varepsilon=$ 
$(c-\max H) / 2>0$. Let $\mathcal{Z}$ be a partition of $[0,1]$ such that $T$ is piecewise monotone with respect to $\mathcal{Z}$ and $\sup _{u, v \in Z}|g(u)-g(v)|<\varepsilon$ for all $Z \in \mathcal{Z}$. Let $\varrho$ be a probability measure with support $A$ which assigns measure zero to single points. We consider the set $N_{d}$ introduced in Section 6 for this partition $\mathcal{Z}$ and this measure $\varrho$. By Lemma 14 there is $d>0$ with $\operatorname{ent}_{\mathrm{B}}\left(N_{d}\right)<\operatorname{ent}_{\mathrm{B}}\left(K_{c}\right)$. In particular, there is $x \in K_{c} \backslash N_{d}$.

Choose $k$ such that $\varrho(Y)<d$ for all $Y \in \mathcal{Z}_{k}$ with $Y \cap A \neq \emptyset$. This is possible since $\varrho$ assigns measure zero to single points and $\mathcal{Z}$ is a generator. The point $x$ chosen above is not an element of $N_{d}$. Hence for all $n \geq 0$ there is $Y_{n} \in \mathcal{Z}_{k}$ with $T^{n}(x) \in Y_{n}$ and there are infinitely many $n$ with $\varrho_{T^{n}(x)}\left(T^{n} Z_{n}(x)\right) \geq d$, which implies $Y_{n} \subset T^{n} Z_{n}(x)$ by the choice of $k$. Since $\mathcal{Z}_{k}$ is finite, we find $C \in \mathcal{Z}_{k}$ and $n_{0}<n_{1}<n_{2}<\cdots$ such that $T^{n_{j}}(x) \in C$ and $C \subset T^{n_{j}} Z_{n_{j}}(x)$ for $j \geq 0$.

Set $y=T^{n_{0}}(x)$ and $m_{j}=n_{j}-n_{0}$ for $j \geq 1$. Because

$$
\begin{aligned}
T^{n_{j}} Z_{n_{j}}(x) & =T^{n_{j}}\left(Z_{n_{0}}(x) \cap T^{-n_{0}} Z_{m_{j}}(y)\right) \\
& =T^{m_{j}}\left(T^{n_{0}} Z_{n_{0}}(x) \cap Z_{m_{j}}(y)\right) \subset T^{m_{j}} Z_{m_{j}}(y)
\end{aligned}
$$

we get $C \subset T^{m_{j}} Z_{m_{j}}(y)$ for $j \geq 1$. Furthermore, for all $j \geq 1$ with $m_{j} \geq k$ we have $Z_{m_{j}}(y) \subset C$ because $y \in C$. For these $j$ we find a point $p_{j}$ in the closure of $Z_{m_{j}}(y)$ which is a fixed point under $T^{m_{j}}$. Let $\nu_{j}$ be the $T$-invariant probability measure on the orbit $B_{j}$ of $p_{j}$. Since $\sup _{u, v \in Z}|g(u)-g(v)|<\varepsilon$ for all $Z \in \mathcal{Z}$ we get $\left|\nu_{j}(g)-\left(1 / m_{j}\right) S_{m_{j}} g(y)\right|<\varepsilon$ for all $j$ large enough, since there can only be finitely many $j$ such that $B_{j}$ contains an endpoint of an interval in $\mathcal{Z}$. This implies

$$
\liminf _{j \rightarrow \infty} \nu_{j}(g) \geq \liminf _{j \rightarrow \infty} \frac{1}{m_{j}} S_{m_{j}} g(y)-\varepsilon=\liminf _{j \rightarrow \infty} \frac{1}{n_{j}} S_{n_{j}} g(x)-\varepsilon \geq c-\varepsilon
$$

where we have also used that $x \in K_{c}$. The function $g$ need not be continuous on the finite set $B_{j}$. But the height of jumps of $g$ at the points of $B_{j}$ tends to zero as $j \rightarrow \infty$. Since the sets $B_{j}$ are Markov subsets of $A$, this implies that the set of limit points of $p\left(T \mid B_{j}, s g\right)$ as $j \rightarrow \infty$ is bounded by $q(T \mid A, s g)=\tau(s)$. Furthermore, $\nu_{j}(s g)=p\left(T \mid B_{j}, s g\right)$ since $B_{j}$ is a periodic orbit. We infer that $s \liminf _{j \rightarrow \infty} \nu_{j}(g) \leq \tau(s)$ for all $s \in \mathbb{R}$, and hence $\liminf _{j \rightarrow \infty} \nu_{j}(g) \in H$ by Proposition 11. By the choice of $\varepsilon$ this contradicts (7.1), and the theorem is proved.

8. The dimension spectrum of Lyapunov exponents. Using similar methods to the last section, we estimate the Hausdorff dimension of level sets of the Lyapunov exponent. Let $A$ be a completely invariant closed subset of $([0,1], T)$ with $h_{\text {top }}(T \mid A)>0$. Suppose that the function $\varphi=\log \left|T^{\prime}\right|$ is regular and set $\tau(s)=q(T \mid A, s \varphi)$. By Lemma 12 there is $s_{0} \in(0,1]$ with $\tau(s) \leq 0$ for $s \leq-s_{0}$ and $\tau(s)>0$ for $s>-s_{0}$. By Proposition 11 the set $H$ is a compact subinterval of $[0, \infty)$ and the set $H^{-1}=\{1 / a: a \in H\}$ 
is a closed subinterval of $(0, \infty)$. Moreover, $a \mapsto \check{\tau}(1 / a)$ is a concave and continuous function on $H^{-1}$, which attains its supremum $s_{0}$ on $H^{-1}$. Therefore the set $\tilde{G}_{0}=\left\{a \in H^{-1}: \check{\tau}(1 / a)=s_{0}\right\}$ is a nonempty closed interval, possibly a single point. Set $\tilde{G}_{+}=\left\{a \in H^{-1}: a<b\right.$ for all $\left.b \in \tilde{G}_{0}\right\}$ and $\tilde{G}_{-}=\left\{a \in H^{-1}: a>b\right.$ for all $\left.b \in \tilde{G}_{0}\right\}$. These sets may be empty. The map $a \mapsto \check{\tau}(1 / a)$ is strictly increasing on $\tilde{G}_{+}$and strictly decreasing on $\tilde{G}_{-}$since it is concave. Finally, set $G_{0}=\left\{1 / a: a \in \tilde{G}_{0}\right\}, G_{+}=\left\{1 / a: a \in \tilde{G}_{+}\right\}$and $G_{-}=\left\{1 / a: a \in \tilde{G}_{-}\right\}$. Then the function $\check{\tau}$ attains its supremum $s_{0}$ on $G_{0}$, is strictly increasing on $G_{-}$and strictly decreasing on $G_{+}$.

For $a \in H$ we define

$$
V_{a}= \begin{cases}\{x \in A: 0<\underline{\chi}(x) \leq \bar{\chi}(x) \leq a\} & \text { if } a \in G_{-}, \\ \{x \in A: \underline{\chi}(x)>0\} & \text { if } a \in G_{0}, \\ \{x \in A: \underline{\chi}(x) \geq a\} & \text { if } a \in G_{+} .\end{cases}
$$

Observe that $0 \notin G_{+}$, since $G_{0}$ is not empty. This implies $\underline{\chi}(x)>0$ for all $x \in V_{a}$ and $a \in H$. Now we can estimate the Hausdorff dimension of these sets. The following proof is similar to the proof of Theorem 16, but details are different.

THEOREM 19. Let $A$ be a completely invariant topologically transitive closed subset of $([0,1], T)$ with $h_{\mathrm{top}}(T \mid A)>0$ and suppose that $\varphi=\log \left|T^{\prime}\right|$ is a regular function. For each $a \in H \backslash\{0\}$ we have $\operatorname{dim}_{\mathrm{H}}\left(V_{a}\right) \leq \check{\tau}(a)$.

Proof. For each $a \in G_{0}$ we have $\check{\tau}(a)=s_{0}$. Since $\tau$ is a convex function, the definition of $\hat{\tau}$ implies that $\check{\tau}(a)=\inf _{s \leq s_{0}}((1 / a) \tau(s)-s)$ if $a \in G_{-}$, and $\check{\tau}(a)=\inf _{s>s_{0}}((1 / a) \tau(s)-s)$ if $a \in G_{+}$.

Fix $a \in H \backslash\{0\}$. Choose $\varepsilon>0$ and $\alpha>0$. Then we can find $s \in \mathbb{R}$ such that $(1 / a) \tau(s)-s<\check{\tau}(a)+\varepsilon$. We can do this in such a way that $s=s_{0}$ if $a \in G_{0}, s \leq s_{0}$ if $a \in G_{-}$, and $s>s_{0}$ if $a \in G_{+}$.

Now choose $\gamma \in(0, \alpha / 2)$ and $\delta>0$ such that $\delta|s| \leq \varepsilon \gamma$ and $\delta|\tau(s)| \leq a \varepsilon \gamma$. Let $\mathcal{Z}$ be a partition of $[0,1]$ with respect to which $T$ is piecewise monotone and with $\operatorname{var}_{\mathcal{Z}}(\varphi)<\min (\delta, \alpha-2 \gamma)$. This is possible, since $\varphi$ is regular. There is a function $\tilde{\varphi}$ which is constant on each interval in $\mathcal{Z}$ and such that $|\tilde{\varphi}-\varphi| \leq \delta$ and $q(T \mid A, s \tilde{\varphi})=q(T \mid A, s \varphi)=\tau(s)$. This is possible by the properties of the pressure function given in Lemma 6 .

By Lemma 8, the notion of pressure we use here is the same as in 9 . Hence we can apply Theorem 2 of [9], which together with Lemma 4 in [7. shows the existence of a probability measure $\varrho$ with support $A$ which is $e^{\tau(s)-s \tilde{\varphi}}$-conformal. This means that $\varrho(T Y)=\int_{Y} e^{\tau(s)-s \tilde{\varphi}} d \varrho$ for all intervals $Y$ contained in elements of $\mathcal{Z}$.

Set

$$
W=\left\{x \in A: \underline{\chi}(x)>0, \liminf _{n \rightarrow \infty} \frac{\tau(s)}{a n} S_{n} \varphi(x) \geq \tau(s)\right\}
$$


We have $V_{a} \subset W$, since $\tau(s)=0$ if $a \in G_{0}, \tau(s) \leq 0$ if $a \in G_{-}$, and $\tau(s)>0$, if $a \in G_{+}$. Let $N_{d}$ and $D_{\alpha}$ be as in Lemma 14. This lemma says that there is $d>0$ with $\operatorname{dim}_{H}\left(N_{d} \cap D_{\alpha}\right)<\varepsilon$, since $\operatorname{var}_{\mathcal{Z}}(\varphi)<\alpha$. Set $W_{\alpha}=\left\{x \in W \backslash N_{d}: \underline{\chi}(x) \geq \alpha\right\}$. We now estimate the Hausdorff dimension of $W_{\alpha}$.

Fix $k \in \mathbb{N}$. The definition of $W$ implies $(\tau(s) / a) S_{n} \varphi(x) \geq n \tau(s)-n \gamma \varepsilon$ for all $x \in W_{\alpha}$ and all but finitely many $n$. Using also the definition of $N_{d}$ and Lemma 13 , for each $x \in W_{\alpha}$ we find an integer $n_{x}$ such that $n_{x} \in I_{d}(x), \quad n_{x} \geq k, \quad \frac{\tau(s)}{a} S_{n_{x}} \varphi(x) \geq n_{x} \tau(s)-\varepsilon \gamma n_{x}, \quad\left|Z_{n_{x}}(x)\right| \leq e^{-\gamma n_{x}}$.

Set $Y_{x}=Z_{n_{x}}(x)$ for $x \in W_{\alpha}$, which is well defined because $W_{\alpha} \subset R_{\mathcal{Z}}$. For $d>0$ found above let $b>0$ be as in Lemma 15. Because $n_{x} \in I_{d}(x)$ we get $\varrho\left(T^{n_{x}} Y_{x}\right) \geq \varrho_{T^{n_{x}(x)}}\left(T^{n_{x}} Y_{x}\right) \geq d$ and $\left|T^{n_{x}} Y_{x}\right| \geq b$ by Lemma 15. Since $\tilde{\varphi}$ is constant on each element of $\mathcal{Z}$, we have $\varrho\left(T^{j+1} Y_{x}\right)=e^{\tau(s)-s \tilde{\varphi}\left(T^{\jmath}(x)\right)} \varrho\left(T^{j} Y_{x}\right)$ for $0 \leq j \leq n_{x}-1$ by the definition of a conformal measure, which implies

$$
\varrho\left(Y_{x}\right)=e^{s S_{n_{x}} \tilde{\varphi}(x)-n_{x} \tau(s)} \varrho\left(T^{n_{x}} Y_{x}\right) \geq d e^{s S_{n_{x}} \tilde{\varphi}(x)-n_{x} \tau(s)} .
$$

Because $\varphi=\log \left|T^{\prime}\right|$ we have $\left|Y_{x}\right| \inf _{Y_{x}} e^{S_{n_{x}} \varphi} \leq\left|T^{n_{x}} Y_{x}\right| \leq\left|Y_{x}\right| \sup _{Y_{x}} e^{S_{n_{x}} \varphi}$. By the definition of $\tilde{\varphi}$ and the choice of $b$, which satisfies $0<b \leq 1$, this implies $e^{s S_{n_{x}} \tilde{\varphi}(x)} \geq b^{|s|}\left|Y_{x}\right|^{-s} e^{-|s| \delta n_{x}}$. By the choice of $\delta$ and $n_{x}$ this gives

$$
e^{s S_{n_{x}} \tilde{\varphi}(x)} \geq b^{|s|}\left|Y_{x}\right|^{-s} e^{-\varepsilon \gamma n_{x}} \geq b^{|s|}\left|Y_{x}\right|^{-s+\varepsilon} .
$$

Similarly we get $e^{-\tau(s) S_{n_{x}} \varphi(x)} \geq b^{|\tau(s)|}\left|Y_{x}\right|^{\tau(s)} e^{-|\tau(s)| \delta n_{x}} \geq b^{|\tau(s)|}\left|Y_{x}\right|^{\tau(s)} e^{-a \varepsilon \gamma n_{x}}$. We have $n_{x} \tau(s) \leq(\tau(s) / a) S_{n_{x}} \varphi(x)+\varepsilon \gamma n_{x}$ and $e^{-\overline{2} \varepsilon \gamma n_{x}} \geq\left|Y_{x}\right|^{2 \varepsilon}$ by the choice of $n_{x}$. The last three inequalities imply

$$
e^{-n_{x} \tau(s)} \geq b^{|\tau(s) / a|}\left|Y_{x}\right|^{\tau(s) / a+2 \varepsilon} .
$$

Putting 8.2 and 8.3 into 8.1 , we get

$$
\varrho\left(Y_{x}\right) \geq d b^{|s|+|\tau(s) / a|}\left|Y_{x}\right|^{\tau(s) / a-s+3 \varepsilon} \quad \text { for all } x \in W_{\alpha} .
$$

Set $\tilde{\mathcal{U}}=\left\{Y_{x}: x \in W_{\alpha}\right\} \subset \bigcup_{j=k}^{\infty} \mathcal{Z}_{j}$. Since two elements of $\bigcup_{j=k}^{\infty} \mathcal{Z}_{j}$ are either disjoint or one contains the other, there is a subset $\mathcal{U}$ of $\tilde{\mathcal{U}}$ which still covers $W_{\alpha}$ and consists of pairwise disjoint intervals. Then (8.4) implies

$$
\sum_{Y \in \mathcal{U}}|Y|^{\tau(s) / a-s+3 \varepsilon} \leq c \sum_{Y \in \mathcal{U}} \varrho(Y) \leq c
$$

where we have written $c$ for $d^{-1} b^{-|s|-|\tau(s) / a|}$. We have chosen $s \in \mathbb{R}$ such that $(1 / a) \tau(s)-s<\check{\tau}(a)+\varepsilon$. This gives $\sum_{Y \in \mathcal{U}}|Y|^{\check{\tau}(a)+4 \varepsilon} \leq c$. By the choice of $n_{x}$ we have $|Y| \leq e^{-\gamma k}$ for all $Y \in \mathcal{U}$, and $k \in \mathbb{N}$ can be chosen arbitrary. It follows that $\nu_{\check{\tau}(a)+4 \varepsilon}\left(W_{\alpha}\right) \leq c$ and hence $\operatorname{dim}_{H}\left(W_{\alpha}\right) \leq \check{\tau}(a)+4 \varepsilon$. This holds for any $\alpha>0$. Furthermore, we have chosen $d$ such that $\operatorname{dim}_{\mathrm{H}}\left(N_{d}\right)<\varepsilon$. 
Because $V_{a} \subset W \subset N_{d} \cup \bigcup_{m=1}^{\infty} W_{1 / m}$ this gives $\operatorname{dim}_{\mathrm{H}}\left(V_{a}\right) \leq \check{\tau}(a)+4 \varepsilon$. Since also $\varepsilon>0$ was arbitrary, this proves $\operatorname{dim}_{\mathrm{H}}\left(V_{a}\right) \leq \check{\tau}(a)$.

For $u, v \in \mathbb{R}$ with $u \leq v$ we now consider the set

$$
M_{u, v}=\{x \in A: u \leq \underline{\chi}(x) \leq \bar{\chi}(x) \leq v\} .
$$

The level set $M_{a}$ which is the set of all $x \in A$ with $\chi(x)=a$ is then the set $M_{a, a}$. We compute the Hausdorff dimension of these sets.

TheOREM 20. Let $A$ be a completely invariant topologically transitive closed subset of $([0,1], T)$ with $h_{\mathrm{top}}(T \mid A)>0$ and suppose that $\varphi=\log \left|T^{\prime}\right|$ is a regular function. For $u$ and $v$ in the interior of $H$ with $u \leq v$ we have $\operatorname{dim}_{\mathrm{H}}\left(M_{u, v}\right)=\max _{a \in[u, v]} \check{\tau}(a)$.

Proof. By Theorem 10, for each $a \in[u, v]$ with $\hat{\tau}(a)>0$ there is an ergodic invariant probability measure $\mu$ on $A$ with $\mu\left(M_{a}\right)=1$ and $h_{\mu} \geq$ $\hat{\tau}(a)$. Because $\mu\left(M_{a}\right)=1$ we have $\operatorname{dim}_{\mathrm{H}}\left(M_{a}\right) \geq h_{\mu} / \mu(\varphi)$ by Lemma 5 and $\mu(\varphi)=a$ by the ergodic theorem. This gives $\operatorname{dim}_{\mathrm{H}}\left(M_{a}\right) \geq h_{\mu} / a \geq \hat{\tau}(a) / a=$ $\check{\tau}(a)$. Because $M_{a} \subset M_{u, v}$ we get $\operatorname{dim}_{\mathrm{H}}\left(M_{u, v}\right) \geq \check{\tau}(a)$, showing $\operatorname{dim}_{\mathrm{H}}\left(M_{u, v}\right) \geq$ $\max _{a \in[u, v]} \check{\tau}(a)$.

In order to show the opposite inequality, we divide $H$ into the sets $G_{-}$, $G_{+}$and $G_{0}$, introduced above. Suppose first that $G_{0} \cap[u, v] \neq \emptyset$. Since $u \in \operatorname{int} H$ and hence $u>0$, we have $M_{u, v} \subset V_{b}$ for some $b \in G_{0} \backslash\{0\}$ by definition of $V_{b}$. We get $\operatorname{dim}_{\mathrm{H}}\left(M_{u, v}\right) \leq \operatorname{dim}_{\mathrm{H}}\left(V_{b}\right) \leq \check{\tau}(b)=\max _{a \in[u, v]} \check{\tau}(a)$ using Theorem 19. If $G_{0} \cap[u, v]=\emptyset$, then either $u \in G_{+}$or $v \in G_{-}$. Furthermore, $0<u \leq v$ since $u \in$ int $H$. If $u \in G_{+}$we get $M_{u, v} \subset V_{u}$, which implies $\operatorname{dim}_{\mathrm{H}}\left(M_{u, v}\right) \leq \operatorname{dim}_{\mathrm{H}}\left(V_{u}\right) \leq \check{\tau}(u) \leq \max _{a \in[u, v]} \check{\tau}(a)$ using Theorem 19. Finally, if $v \in G_{-}$we get $M_{u, v} \subset V_{v}$, which implies $\operatorname{dim}_{\mathrm{H}}\left(M_{u, v}\right) \leq \operatorname{dim}_{\mathrm{H}}\left(V_{v}\right) \leq$ $\check{\tau}(v) \leq \max _{a \in[u, v]} \check{\tau}(a)$ again by Theorem 19 .

\section{References}

[1] L. Barreira, Y. Pesin and J. Schmeling, On a general concept of multifractality: Multifractal spectra for dimensions, entropies, and Lyapunov exponents. Multifractal rigidity, Chaos 7 (1997), 27-38.

[2] L. Barreira and B. Saussol, Variational principles and mixed multifractal spectra, Trans. Amer. Math. Soc. 353 (2001), 3919-3944.

[3] R. Bowen, Topological entropy for noncompact sets, ibid. 184 (1973), 125-136.

[4] K. Gelfert and M. Rams, The Lyapunov spectrum of some parabolic systems, Ergodic Theory Dynam. Systems 29 (2009), 919-940.

[5] F. Hofbauer, Piecewise invertible dynamical systems, Probab. Theory Related Fields 72 (1986), 359-368.

[6] - , An inequality for the Lyapunov exponent of an ergodic invariant measure for a piecewise monotone map of the interval, in: Lyapunov Exponents (Oberwolfach, 1990), L. Arnold et al. (eds.), Lecture Notes in Math. 1486, Springer, Berlin, 1991, $227-231$. 
[7] F. Hofbauer, Local dimension for piecewise monotonic maps on the interval, Ergodic Theory Dynam. Systems 15 (1995), 1119-1142.

[8] F. Hofbauer and P. Raith The Hausdorff dimension of an ergodic invariant measure for a piecewise monotonic map of the interval, Canad. Math. Bull. 35 (1991), 1-15.

[9] F. Hofbauer and M. Urbański, Fractal properties of invariant subsets for piecewise monotonic maps on the interval, Trans. Amer. Math. Soc. 343 (1994), 659-673.

[10] W. Parry, Intrinsic Markov chains, ibid. 112 (1964), 55-66.

[11] Y. Pesin, Dimension Theory in Dynamical Systems: Contemporary Views and Applications, Chicago Lectures in Mathematics, The Univ. of Chicago Press, 1997

[12] Y. Pesin and B. Pitskel, Topological pressure and the variational principle for noncompact sets, Funct. Anal. Appl. 18 (1984), 307-318.

[13] Y. Pesin and H. Weiss, A multifractal analysis of Gibbs measures for conformal expanding maps and Markov Moran geometric constructions, J. Statist. Phys. 86 (1997), 233- 275.

[14] F. Takens and E. Verbitsky Multifractal analysis of local entropies for expansive homeomorphisms with specification, Comm. Math. Phys. 203 (1999), 593-612.

[15] - - - On the variational principle for the topological entropy of certain noncompact sets, Ergodic Theory Dynam. Systems 23 (2003), 317-348.

[16] P. Walters, A variational principle for the pressure of continuous transformations, Amer. J. Math. 97 (1976), 931-971.

[17] —, An Introduction to Ergodic Theory, Springer, Berlin, 1982.

Franz Hofbauer

Institut für Mathematik

Universität Wien

Nordbergstrasse 15

1090 Wien, Austria

E-mail: franz.hofbauer@univie.ac.at

Received 7 October 2008;

in revised form 9 December 2009 